\title{
A Generalized Topological Degree in Admissible Linear Spaces
}

\author{
T. Okon
}

\begin{abstract}
The paper deals with the construction of a degree which extends the Generalized Topological Degree of Browder and Petryshyn to admissible linear spaces introducing the notion of approximation nets and approximation-compact maps. After discussing the usual properties of a degree the relationship between approximation compactness and the concept of condensing maps is analysed. The non-locally convex spaces $l^{p}, H^{p}, N^{+}, L_{p}[0,1]$ and $S[0,1](0<p<1)$ are considered for illustration. The main results are fixed-point theorems for this spaces. Concrete examples of operators are given for $l^{p}$ and $H^{p}$.
\end{abstract}

Keywords: Admissible spaces, topological degree, condensing maps

AMS subject classification: Primary $47 \mathrm{H} 11$, secondary 47 H 09, 47 H 10

\section{Introduction}

The Leray-Schauder degree for compact perturbations of the identity has been generalized in several directions. The aim of this paper is to join two such directions by constructing a more general degree.

Sadovskii [15] has developed a degree for limit-compact operators in locally convex spaces. Operators which condense with respect to a measure of non-compactness are typical examples of limit-compact operators.

Kaballo [6] has defined a degree for compact perturbations of the identity in admissible topological vector spaces which are not necessary locally convex (see Subsection 2.1).

The question of joining these developments appears in the paper of Kayser [7]: What about a degree for condensing operators in admissible topological vector spaces? Analysing this question, a problem arises which only at first sight is a technical one. Using the classical way to construct a degree for condensing operators, a retract on a set called limit range of the operator must exist. It turns out that the convexity of this set is not only of topological necessity and the classical construction cannot be used. The relationship between retracts and admissibility has been analysed in [17] and [18]. Up to now it is unknown if there exists a topological vector space which is not admissible.

The last result concerning Kayser's question is the paper of Alex and co-workers [1], where a degree for compact reducible operators in non-locally convex spaces is defined.

T. Okon: Universität Dortmund, FB Mathematik (am Lehrstuhl 1), D - 44221 Dortmund 
Alex and his co-workers use the notion of locally convex subsets in topological vector spaces.

Our access to this problem has been motivated by the paper of Browder and Petryshyn [2]. The construction is free of convexity and turns out a set-valued degree which extends that of [6]. Our notion of measures of non-compactness and condensing operators is free of convexity, too. So, we get a more general class of condensing operators but the new degree is not defined for all of them. The question whether or not the new degree solves the problem of Kayser is given to the reader.

\section{Construction of the degree}

2.1 Approximation nets and approximation-compact maps. Let $X$ be a Hausdorff topological vector space over the scalar field $I R$ and $\mathcal{U}$ the system of all circled neighbourhoods of the origin in $X$. The space $X$ is called admissible provided that for every compact set $K \subseteq X$ and every neighbourhood $U \in \mathcal{U}$ we can find a finite-dimensional subspace $X^{\prime}$ of $X$ and a continuous map $I^{\prime}: K \rightarrow X^{\prime}$ such that $\left(I-I^{\prime}\right)(K) \subseteq U$, where $I$ denotes the identity. Since $X$ is a uniform space and $K$ is compact we can extend $I^{\prime}$ to a compact map defined on the whole space $X$ by making use of the construction which leads to the Tietze-Urysohn extension theorem. Therefore, without loss of generality, let $I^{\prime}$ be defined on $X$.

There is a natural partial ordering on these maps: Let $\mathcal{K}$ be the system of all compact subsets of $X$. For $\left(K_{i}, U_{i}\right) \in \mathcal{K} \times \mathcal{U}(i=1,2)$ we define

$$
\left(K_{1}, U_{1}\right) \geq\left(K_{2}, U_{2}\right) \quad \Longleftrightarrow \quad K_{1} \supseteq K_{2} \text { and } U_{1} \subseteq U_{2} \text {. }
$$

By choosing such a map $I^{\prime}$ for every pair $(K, U) \in \mathcal{K} \times \mathcal{U}$ we get a directed system of approximations. This system motivates the notion of an approximation net we now introduce.

Definition 1. Let $X$ be an admissible space and $(\Gamma, \leq)$ a directed system. For every $\gamma \in \Gamma$ assume that finite-dimensional subspaces $X_{\gamma} \subseteq X$ and continuous maps $P_{\gamma}: X \rightarrow X_{\gamma}$ are given such that, for all $K \subseteq X$ compact and for all $U \in \mathcal{U}$, there exists a $\gamma_{0} \in \Gamma$ such that

$$
\left(I-P_{\gamma}\right)(K) \subseteq U \quad \text { for all } \gamma \geq \gamma_{0} .
$$

The system $\left(P_{\gamma}\right)_{\gamma \in \Gamma}$ is called an approximation net.

In the following the notions linear approximation net and approximation sequence are used if the maps $P_{\gamma}$ are linear or $\Gamma$ is countable, respectively.

In case of a Banach space $X$ and $\Gamma=I N$ we get an approximation scheme

$$
\left(\left\{X_{n}\right\},\left\{X_{n}\right\},\left\{I_{n}\right\},\left\{P_{n}\right\}\right)
$$

in the sense of Browder and Petryshyn [2], at which $I_{n}: X_{n} \rightarrow X$ denotes the embedding of $X_{n}$ into $X$.

Now an analogon of the $A$-proper maps of [2] will be defined. 
Definition 2. Let $\emptyset \neq \Omega \subseteq X$ be open and $\emptyset \neq Y \subseteq X$. Let $\left(P_{\gamma}\right)_{\gamma \in \Gamma}$ be an approximation net in $X$ and $\Omega_{\gamma}:=\Omega \cap X_{\gamma} \quad(\gamma \in \Gamma)$. We call a continuous map $F: \bar{\Omega} \rightarrow X$ approximation-compact with respect to $Y$ and $\left(P_{\gamma}\right)_{\gamma \in \Gamma}$ if for any $y \in Y$, subnet $\Gamma^{\prime}$ of $\Gamma$ and system $\left(x_{\gamma^{\prime}}\right)_{\gamma^{\prime} \in \Gamma^{\prime}} \subseteq X$ the following property is fulfilled:

(A) If for all $\gamma^{\prime} \in \Gamma^{\prime}$

$$
x_{\gamma^{\prime}} \in \bar{\Omega}_{\gamma^{\prime}} \quad \text { and } \quad \lim _{\gamma^{\prime}}\left(x_{\gamma^{\prime}}-P_{\gamma^{\prime}} F x_{\gamma^{\prime}}\right)=y
$$

then there exist a subnet $\Gamma^{\prime \prime}$ of $\Gamma^{\prime}$ and an element $x \in \bar{\Omega}$ such that

$$
\lim _{\gamma^{\prime \prime}} x_{\gamma^{\prime \prime}}=x \quad \text { and } \quad x-F x=y .
$$

Let us agree on some notations again: $\mathrm{By} \Omega_{\gamma}$ and $X_{\gamma}$ we always denote the sets in the preceding definitions. In case of $Y=X$ or $Y=\{y\}$ we call $F$ approximation-compact or approximation-compact with respect to $y$, respectively.

The following theorem is a first hint that the degree, which will be defined in Subsection 2.2, is an extension of Kaballo's degree which is defined for compact perturbations of the identity (see [6]). It is also a first example of an approximation-compact map.

Theorem 1. Let $\left(P_{\gamma}\right)_{\gamma \in \Gamma}$ be an approximation net in $X$ and $F: \bar{\Omega} \rightarrow X$ a continuous and compact map, where $\Omega \subseteq X$ is open. Then $F$ is approximation-compact.

Proof. Let $\left(x_{\gamma^{\prime}}\right)_{\gamma^{\prime} \in \Gamma^{\prime}}$ be as in assumption (A). Thus, we have for a fixed $y \in X$

$$
\lim _{\gamma^{\prime}}\left(x_{\gamma^{\prime}}-P_{\gamma^{\prime}} F x_{\gamma^{\prime}}\right)=y
$$

Since $\left(P_{\gamma^{\prime}}\right)_{\gamma^{\prime} \in \Gamma^{\prime}}$ is an approximation net, for any $U \in \mathcal{U}$ there exists a $\gamma^{\prime} \in \Gamma^{\prime}$ such that

$$
\left(I-P_{\hat{\gamma}}\right)(F(\bar{\Omega})) \subseteq U \quad \text { for all } \hat{\gamma} \geq \gamma^{\prime}
$$

Relations (1) and (2) lead to

$$
\lim _{\boldsymbol{\gamma}^{\prime}}\left(x_{\boldsymbol{\gamma}^{\prime}}-F \boldsymbol{x}_{\boldsymbol{\gamma}^{\prime}}\right)=\boldsymbol{y}
$$

Because of the relative compactness of $\left\{F\left(x_{\gamma^{\prime}}\right) \mid \gamma^{\prime} \in \Gamma^{\prime}\right\}$, there exists a subnet $\Gamma^{\prime \prime}$ of $\Gamma^{\prime}$ such that $\lim _{\gamma^{\prime \prime}} F x_{\gamma^{\prime \prime}}$ exists. Using (3) we get the existence of an $x \in \bar{\Omega}$ with $\lim _{\gamma^{\prime \prime}} x_{\gamma^{\prime \prime}}=x$. Finally $x-F x=y$ follows from the continuity of $F$

The following lemma deals with a separation property, which is a technical necessity for constructing a degree.

Lemma 1. Suppose $\emptyset \neq \Omega \subseteq X$ is open and $\left(P_{\gamma}\right)_{\gamma \in \Gamma}$ is an approximation net in $X$. Let the function $F: \bar{\Omega} \rightarrow X$ be approximation-compact with respect to $y \in$ $X \backslash(I-F)(A)$, where $A \subseteq \bar{\Omega}$ is closed. Then there exist $U_{0} \in \mathcal{U}$ and $\gamma_{0} \in \Gamma$ such that

$$
P_{\gamma} y \notin\left(I-P_{\gamma} F\right)\left(A \cap \bar{\Omega}_{\gamma}\right)+U_{0}
$$


for every $\gamma \geq \gamma_{0}$.

Proof. If the statement is not true, then for every $\gamma \in \Gamma$ and $U \in \mathcal{U}$ there exist $\gamma^{\prime}=\gamma^{\prime}(\gamma, U) \geq \gamma$ and $x_{\gamma^{\prime}} \in A \cap \bar{\Omega}_{\gamma^{\prime}}$ such that

$$
x_{\gamma^{\prime}}-P_{\gamma^{\prime}} F x_{\gamma^{\prime}}-P_{\gamma^{\prime}} y \in U \text {. }
$$

By choosing such a $\gamma^{\prime}$ for every pair $(\gamma, U) \in \Gamma \times \mathcal{U}$ a subnet $\Gamma^{\prime}$ of $\Gamma$ is defined by the partial ordering

$$
\gamma_{1}^{\prime}=\gamma_{1}^{\prime}\left(\gamma_{1}, U_{1}\right) \geq \gamma_{2}^{\prime}\left(\gamma_{2}, U_{2}\right)=\gamma_{2}^{\prime} \quad \Longleftrightarrow \quad \gamma_{1} \geq \gamma_{2} \text { and } U_{1} \subseteq U_{2}
$$

Assumption (A) holds for $\left(x_{\gamma^{\prime}}\right)_{\gamma^{\prime} \in \Gamma^{\prime}}$ and the approximation compactness of $F$ provides a subnet $\Gamma^{\prime \prime}$ of $\Gamma^{\prime}$ and an element $x \in A$ with $\lim _{\gamma^{\prime \prime}} x_{\gamma^{\prime \prime}}=x \in A$ and $x-F x=y$. But this contradicts $y \in X \backslash(I-F)(A)$

2.2 Definition and properties of the generalized degree. In this subsection $\emptyset \neq \Omega \subseteq X$ is always open and $\left(P_{\gamma}\right)_{\gamma \in \Gamma}$ denotes an approximation net in $X$.

Since $\Omega$ is open, there exists a $\gamma_{1} \in \Gamma$ such that $\Omega_{\gamma} \neq \emptyset$ for $\gamma \geq \gamma_{1}$. Let $F: \bar{\Omega} \rightarrow X$

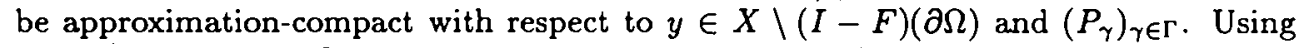
Lemma 1 with $A=\partial \Omega$ we get a $\gamma_{2} \in \Gamma$ such that $P_{\gamma} y \notin\left(I-P_{\gamma} F\right)\left(\partial \Omega_{\gamma}\right)$ for $\gamma \geq \gamma_{2}$.

If we suppose the existence of a $\gamma_{3} \in \Gamma$ such that

$$
\left(I-P_{\gamma} F\right)^{-1}\left(P_{\gamma} y\right) \quad \text { is bounded for every } \gamma \geq \gamma_{3},
$$

then the Brouwer degree

$$
\operatorname{deg}_{n_{\gamma}}\left(I-P_{\gamma} F, \Omega_{\gamma}, P_{\gamma} y\right)
$$

is defined in the finite-dimensional space $X_{\gamma}$. Here, $n_{\gamma}$ denotes the dimension of the space $X_{\gamma}$.

Definition 3. Let $F: \bar{\Omega} \rightarrow X$ be approximation-compact with respect to $y \in$

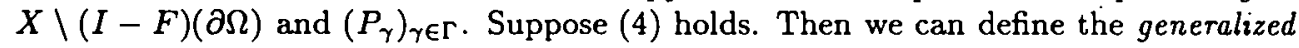
degree of $I-F$ with respect to $y$

$$
\operatorname{deg}(I-F, \Omega, y)
$$

by the set of all cluster points of the net

$$
\left(\operatorname{deg}_{n_{\gamma}}\left(I-P_{\gamma} F, \Omega_{\gamma}, P_{\gamma} y\right)\right)_{\gamma \geq \gamma_{1}, \gamma_{2}, \gamma_{3}}
$$

in $\mathbb{Z} \cup\{ \pm \infty\}$.

Since the definition of $\operatorname{deg}(I-F, \Omega, y)$ is independent of $\gamma_{1}, \gamma_{2}$ and $\gamma_{3}$ we can write $\Gamma$ instead of $\gamma \geq \gamma_{1}, \gamma_{2}, \gamma_{3}$ if (4) holds.

Condition (4) is not as restrictive as one might think. For instance, if the maps $P_{\gamma}$ are compact or $F$ is compact, then (4) holds. Especially the natural net, given by the Tietze-Urysohn extension theorem, can be used to define the degree. In case of a linear approximation net the compactness of $F$ can be replaced by boundedness since continuous linear maps map bounded sets into bounded sets.

The next theorem shows that in contrast to the requirements on $F$ we can get (4) also in a category of spaces. 
Theorem 2. Let $X$ be a locally bounded space and $\left(P_{n}\right)_{n \in \mathbb{N}}$ an approximation sequence in $X$. Suppose $F: \bar{\Omega} \rightarrow X$ is approximation-compact with respect to $y \in X$ and $\left(P_{n}\right)_{n \in \mathbb{N}}$. Then there exists a $\gamma_{3}=n_{3} \in \mathbb{N}$ providing (4).

Proof. Otherwise, we put $A_{n}=\left(I-P_{n} F\right)^{-1}\left(P_{n} y\right)$ for $n \in N$ and get a sequence $\left(A_{n_{k}}\right)_{k \in \mathbb{N}}$ of unbounded sets. Let $p_{U}$ name the Minkowski functional, generated by a bounded $U \in \mathcal{U}$. Since $p_{U}$ is unbounded on every $A_{n_{k}}$ we can choose $x_{n_{k}} \in A_{n_{k}}$ with $p_{U}\left(x_{n_{k}}\right) \geq k$ for every $k \in \mathbb{N}$. The sequence $\left(x_{n_{k}}\right)_{k \in \mathbb{N}}$ satisfies assumption (A), but $\left(x_{n_{k}}\right)_{k \in \mathbb{N}}$ has no convergent subsequence. This contradicts to the approximation compactness of $F$

Next we prove the characteristic properties of a topological degree followed by a result of "Borsuk type".

Theorem 3. Let $F: \bar{\Omega} \rightarrow X$ be approximation-compact with respect to $y \in X \backslash$ $(I-F)(\partial \Omega)$ and $\left(P_{\gamma}\right)_{\gamma \in \Gamma}$. Then we have

(i) $\operatorname{deg}(I-F, \Omega, y) \neq \emptyset$,

(ii) $\operatorname{deg}(I-F, \Omega, y) \neq\{0\}$ implies the existence of some $x \in \Omega$ with $x-F x=y$

and the normalization of the degree turns out as

(G1) $\operatorname{deg}(I, \Omega, y)= \begin{cases}\{1\} & \text { if } y \in \Omega \\ \{0\} & \text { if } y \in X \backslash \bar{\Omega} .\end{cases}$

Proof. (i) holds, since the topological space $\mathbb{Z} \cup\{ \pm \infty\}$ is compact. Concerning (ii) we first notice that $\operatorname{deg}(I-F, \Omega, y) \neq\{0\}$ implies the existence of a subnet $\Gamma^{\prime}$ of $\Gamma$ such that $\operatorname{deg}_{n_{\gamma^{\prime}}}\left(I-P_{\gamma^{\prime}} F, \Omega_{\gamma^{\prime}}, P_{\gamma^{\prime}} y\right) \neq 0$ holds for all $\gamma^{\prime} \in \Gamma^{\prime}$. The property of the Brouwer degree which corresponds to (ii) provides $x_{\gamma^{\prime}} \in \Omega_{\gamma^{\prime}}$ with $x_{\gamma^{\prime}}-P_{\gamma^{\prime}} F_{x_{\gamma^{\prime}}}=P_{\gamma^{\prime}} y$ for all $\gamma^{\prime} \in \Gamma^{\prime}$. Now the assertion follows directly from the approximation compactness of $F$ noting that $\left(P_{\gamma^{\prime}}\right)_{\gamma^{\prime} \in \Gamma^{\prime}}$ is also an approximation net and $y \notin(I-F)(\partial \Omega)$ holds.

To prove the normalization (G1) we first handle the case $y \in \Omega$ : After choosing $U \in \mathcal{U}$ with $y+U+U \subseteq \Omega$ we can get $\gamma_{0} \in \Gamma$ such that $P_{\gamma} y \in y+U$ and $P_{\gamma} 0 \in U$ hold for all $\gamma \geq \gamma_{0}$. Now define

$$
H_{\gamma}(t, x)=x-t P_{\gamma} 0 \quad\left(x \in \bar{\Omega}_{\gamma}, t \in[0,1], \gamma \geq \gamma_{0}\right) .
$$

Since $U$ is circled, we have $H_{\gamma}(t, x) \in x+U$ for $x \in \partial \Omega_{\gamma}, t \in[0,1]$ and $\gamma \geq \gamma_{0}$. That means $H_{\gamma}(t, x) \neq P_{\gamma} y$ for $x \in \partial \Omega_{\gamma}, t \in[0,1]$ and $\gamma \geq \gamma_{0}$. Hence, the homotopy invariance of the Brouwer degree implies

$$
\operatorname{deg}_{n_{\gamma}}\left(I-P_{\gamma} 0, \Omega_{\gamma}, P_{\gamma} y\right)=\operatorname{deg}_{n_{\gamma}}\left(I, \Omega_{\gamma}, P_{\gamma} y\right) \quad\left(\gamma \geq \gamma_{0}\right)
$$

Property (5) and the normalization of the Brouwer degree provide

$$
\operatorname{deg}_{n_{\gamma}}\left(I-P_{\gamma} 0, \Omega_{\gamma}, P_{\gamma} y\right)=1 \quad\left(\gamma \geq \gamma_{0}\right)
$$

Since $(\gamma)_{\gamma \geq \gamma_{0}}$ is a subnet of $\Gamma$ and, on the other hand, every subnet of $\Gamma$ and $(\gamma)_{\gamma \geq \gamma_{0}}$ have a subnet in common we get $\operatorname{deg}(I, \Omega, y)=\{1\}$.

For $y \in X \backslash \bar{\Omega}, \operatorname{deg}(I-\Omega, y)=\{0\}$ can be shown in the same way 
Theorem 4. Let $\emptyset \neq \Omega_{1}, \Omega_{2} \subseteq \Omega$ be open and disjoint. Suppose $F: \bar{\Omega} \rightarrow X$ is approximation-compact with respect to $y \in X \backslash(I-F)\left(\bar{\Omega} \backslash\left(\Omega_{1} \cup \Omega_{2}\right)\right)$ and $\left(P_{\gamma}\right)_{\gamma \in \Gamma}$. Then the restrictions $F: \bar{\Omega}_{i} \rightarrow X(i=1,2)$ are approximation-compact with respect to $y$ and $\left(P_{\gamma}\right)_{\gamma \in \Gamma}$, and we have

(G2) $\operatorname{deg}(I-F, \Omega, y) \subseteq \operatorname{deg}\left(I-F, \Omega_{1}, y\right)+\operatorname{deg}\left(I-F, \Omega_{2}, y\right)$

under the convention that $\infty+(-\infty)=z$ holds for every $z \in \mathbb{Z} \cup\{ \pm \infty\}$.

If there exist $i \in\{1,2\}$ and $z \in \mathbb{Z}$ such that $\operatorname{deg}\left(I-F, \Omega_{i}, y\right)=\{z\}$, we have ' = ' instead of ' $\subseteq$ ' in (G2).

Proof. Since $\bar{\Omega} \backslash\left(\Omega_{1} \cup \Omega_{2}\right)$ is closed, there exists a $\gamma_{0} \in \Gamma$ such that

$$
P_{\gamma} y \notin\left(I-P_{\gamma} F\right)\left(\left(\bar{\Omega} \backslash\left(\Omega_{1} \cup \Omega_{2}\right)\right) \cap X_{\gamma}\right) \quad \text { for } \gamma \geq \gamma_{0}
$$

Now we can deal with the finite-dimensional case. The property of the Brouwer degree which corresponds to (G2) provides

$$
\begin{aligned}
& \operatorname{deg}_{n_{\gamma}}\left(I-P_{\gamma} F, \Omega_{\gamma}, P_{\gamma} y\right) \\
& \quad=\operatorname{deg}_{n_{\gamma}}\left(I-P_{\gamma} F, \Omega_{1_{\gamma}}, P_{\gamma} y\right)+\operatorname{deg}_{n_{\gamma}}\left(I-P_{\gamma} F, \Omega_{2_{\gamma}}, P_{\gamma} y\right) \quad\left(\gamma \geq \gamma_{0}\right) .
\end{aligned}
$$

If $\operatorname{deg}(I-F, \Omega, y) \cap \mathbb{Z} \neq \emptyset$ we choose $z \in \operatorname{deg}(I-F, \Omega, y) \cap \mathbb{Z}$. There exists a subnet $\Gamma^{\prime}$ of $\Gamma$ such that $\operatorname{deg}_{n_{\gamma^{\prime}}}\left(I-P_{\gamma^{\prime}} F, \Omega_{\gamma^{\prime}}, P_{\gamma^{\prime}} y\right)=z$ for $\gamma^{\prime} \in \Gamma^{\prime}$.

If there exists a subnet

$$
\left(\operatorname { d e g } _ { n _ { \gamma ^ { \prime \prime } } } ( I - P _ { \gamma ^ { \prime \prime } } F , \Omega _ { 1 _ { \gamma ^ { \prime \prime } } } , P _ { \gamma ^ { \prime \prime } } y ) _ { \gamma ^ { \prime \prime } \in \Gamma ^ { \prime \prime } } \text { of } \left(\operatorname{deg}_{n_{\gamma^{\prime}}}\left(I-P_{\gamma^{\prime}} F, \Omega_{1_{\gamma^{\prime}}}, P_{\gamma^{\prime}} y\right)_{\gamma^{\prime} \in \Gamma^{\prime}}\right.\right.
$$

converging to $z^{\prime} \in \mathbb{Z}$ we obtain, using (6), that

$$
\left(\operatorname{deg}_{n_{\gamma^{\prime \prime}}}\left(I-P_{\gamma^{\prime \prime}} F, \Omega_{\gamma_{\gamma^{\prime \prime}}}, P_{\gamma^{\prime \prime}} y\right)_{\gamma^{\prime \prime} \in \Gamma^{\prime \prime}} \quad \text { converges to } z-z^{\prime}\right.
$$

If there is no such subnet, Theorem $3 /(\mathrm{i})$ guarantees the existence of a subnet

$$
\left(\operatorname{deg}_{n_{\gamma^{\prime \prime}}}\left(I-P_{\gamma^{\prime \prime}} F, \Omega_{1_{\gamma^{\prime \prime}}}, P_{\gamma^{\prime \prime}} y\right)_{\gamma^{\prime \prime} \in \Gamma^{\prime \prime}} \quad \text { converging to } \pm \infty\right. \text {, }
$$

respectively. Again, using (6),

$$
\left(\operatorname{deg}_{n_{\gamma^{\prime \prime}}}\left(I-P_{\gamma^{\prime \prime}} F, \Omega_{2_{\gamma^{\prime \prime}}}, P_{\gamma^{\prime \prime}} y\right)_{\gamma^{\prime \prime} \in \Gamma^{\prime \prime}} \text { converges to } \pm \infty\right.
$$

respectively.

In case of $\pm \infty \in \operatorname{deg}(I-F, \Omega, y)$ the argumentation is analogous.

To prove the equality statement we only note that, for example, $\operatorname{deg}(I-F, \Omega, y)=$ $\{z\} \subseteq \mathbb{Z}$ provides the existence of a $\gamma_{0} \in \Gamma$ such that $\operatorname{deg}_{n_{\gamma}}\left(I-P_{\gamma} F, \Omega_{\gamma}, P_{\gamma}\right)=z$ for $\gamma \geq \gamma_{0}$ 
In contrast to the classical degrees - for example the Leray-Schauder degree - our degree bases on a family of approximations. The effect on the homotopy invariance is that we have to assume "stronger continuity" than in the classical case.

Theorem 5. Let $F:[0,1] \times \bar{\Omega} \rightarrow X$ and $y:[0,1] \rightarrow X$ be continuous. Suppose that $F(t, \cdot)$ is approximation-compact with respect to $y(t)$ and $\left(P_{\gamma}\right)_{\gamma \in \Gamma}$ for every $t \in[0,1]$. Furthermore, suppose the following three conditions:

(i) The map $t \mapsto F(t, \cdot)$ is continuous with respect to the topology of uniform convergence on $\bar{\Omega}$.

(ii) $y(t) \neq x-F(t, x)$ holds for $t \in[0,1]$ and $x \in \partial \Omega$.

(iii) For $\left(a_{\gamma}\right)_{\gamma \in \Gamma},\left(b_{\gamma}\right)_{\gamma \in \Gamma} \subseteq F([0,1] \times \bar{\Omega})$ with $\lim _{\gamma}\left(a_{\gamma}-b_{\gamma}\right)=0$ the relation $\lim _{\gamma}\left(P_{\gamma} a_{\gamma}-P_{\gamma} b_{\gamma}\right)=0$ holds.

Then $\operatorname{deg}(I-F(t, \cdot), \Omega, y(t))$ is independent of $t \in[0,1]$.

Proof. Referring to the homotopy invariance of the finite-dimensional Brouwer degree it is sufficient to show the existence of a $\gamma_{0} \in \Gamma$ such that

$$
P_{\gamma} y(t) \notin\left(I-P_{\gamma} F(t, \cdot)\right)\left(\partial \Omega_{\gamma}\right) \quad \text { for } t \in[0,1], \gamma \geq \gamma_{0}
$$

Otherwise, for any $\gamma \in \Gamma$ there exist $\gamma^{\prime} \geq \gamma, t_{\gamma^{\prime}} \in[0,1]$ and $x_{\gamma^{\prime}} \in \partial \Omega_{\gamma^{\prime}}$ such that

$$
P_{\gamma^{\prime}} y\left(t_{\gamma^{\prime}}\right)=x_{\gamma^{\prime}}-P_{\gamma^{\prime}} F\left(t_{\gamma^{\prime}}, x_{\gamma^{\prime}}\right) \text {. }
$$

Choosing such a $\gamma^{\prime}$ for every $\gamma \in \Gamma$ a subnet $\Gamma^{\prime}$ of $\Gamma$ is defined. Without loss of generality we can assume the convergence of $\left(t_{\gamma^{\prime}}\right)_{\gamma^{\prime} \in \Gamma^{\prime}}$ to a $t \in[0,1]$. Using (i) we have

$$
\lim _{\gamma^{\prime}}\left(F\left(t_{\gamma^{\prime}}, x_{\gamma^{\prime}}\right)-F\left(t, x_{\gamma^{\prime}}\right)\right)=0
$$

Condition (iii) holds for all subnets $\Gamma^{\prime}$ of $\Gamma-$ fill up $\left(a_{\gamma^{\prime}}\right)_{\gamma^{\prime} \in \Gamma^{\prime}}$ and $\left(b_{\gamma^{\prime}}\right)_{\gamma^{\prime} \in \Gamma^{\prime}}$ with zeros. Therefore,

$$
\lim _{\gamma^{\prime}}\left(P_{\gamma} F\left(t_{\gamma^{\prime}}, x_{\gamma^{\prime}}\right)-P_{\gamma} F\left(t, x_{\gamma^{\prime}}\right)\right)=0
$$

Using that $\left(P_{\gamma^{\prime}}\right)_{\gamma^{\prime} \in \Gamma^{\prime}}$ is also an approximation net we have

$$
\lim _{\gamma^{\prime}} P_{\gamma^{\prime}} y\left(t_{\gamma^{\prime}}\right)=y(t)
$$

since $\left\{y\left(t_{\gamma^{\prime}}\right) \mid \gamma^{\prime} \in \Gamma^{\prime}\right\}$ is relatively compact and $y$ is continuous. Together, (7) - (9) yield

$$
\lim _{\gamma^{\prime}}\left(x_{\gamma^{\prime}}-P_{\gamma^{\prime}} F\left(t_{\gamma^{\prime}}, x_{\gamma^{\prime}}\right)\right)=y(t)
$$

Since $F$ is approximation-compact, there exist a subnet $\Gamma^{\prime \prime}$ of $\Gamma^{\prime}$ and an $x \in \bar{\Omega}$ with $\lim _{\gamma^{\prime \prime}} x_{\gamma^{\prime \prime}}=x$ and $x-F x=y$. Since $x_{\gamma^{\prime \prime}} \in \partial \Omega_{\gamma^{\prime \prime}} \subseteq \partial \Omega$ holds for all $\gamma^{\prime \prime} \in \Gamma^{\prime \prime}$, we have the contradiction $x \in \partial \Omega$ 
Clearly, condition (iii) of Theorem 5 is a purely technical one. For example, it holds for a uniformly equicontinuous approximation net.

Our last theorem is of "Borsuk type".

Theorem 6. Let $0 \in \Omega$ for an $\Omega$ which is symmetric with respect to the origin, i.e. $\Omega=-\Omega$. Suppose $F: \bar{\Omega} \rightarrow X$ is odd, i.e. $F(-x)=-F(x)$ for $x \in \bar{\Omega}$. Suppose further that $F: \bar{\Omega} \rightarrow X$ is approximation-compact with respect to 0 and $\left(P_{\gamma}\right)_{\gamma \in \Gamma}$. Finally let $\left(P_{\gamma}\right)_{\gamma \in \Gamma}$ consist of odd mappings and $0 \notin(I-F)(\partial \Omega)$. Then $\operatorname{deg}(I-F, \Omega, y)$ is odd, i.e. $\operatorname{deg}(I-F, \Omega, y) \cap 2 \mathbb{Z}=\emptyset$.

Proof. Fix $\gamma \in \Gamma$. The set $\Omega_{\gamma}$ is symmetric with respect to the origin, $0 \in \Omega_{\gamma}$ holds and the map $I-P_{\gamma} F$ is odd. So, using the finite-dimensional Borsuk theorem,

$$
\operatorname{deg}\left(I-P_{\gamma} F, \Omega_{\gamma}, 0\right) \quad \text { is odd if } \quad 0 \notin\left(I-P_{\gamma} F\right)\left(\partial \Omega_{\gamma}\right) .
$$

Therefore, it is sufficient to show the existence of a $\gamma_{0} \in \Gamma$ such that $\operatorname{deg}_{n_{\gamma}}\left(I-P_{\gamma} F, \Omega_{\gamma}, 0\right)$ is defined and

$$
\operatorname{deg}_{n_{\gamma}}\left(I-P_{\gamma} F, \Omega_{\gamma}, P_{\gamma} 0\right)=\operatorname{deg}_{n_{\gamma}}\left(I-P_{\gamma} F, \Omega_{\gamma}, 0\right) \quad \text { for } \gamma \geq \gamma_{0} .
$$

We apply Lemma 1 with $A=\partial \Omega$. Since $\partial \Omega_{\gamma} \subseteq \partial \Omega(\gamma \in \Gamma)$, we have the existence of some $\gamma_{1} \in \Gamma$ and $U_{0} \in \mathcal{U}$ such that

$$
\left(I-P_{\gamma} F\right)\left(\partial \Omega_{\gamma}\right) \cap\left(P_{\gamma} 0+U_{0}\right)=\emptyset \quad \text { for } \gamma \geq \gamma_{1}
$$

Further on, noting $\lim _{\gamma} P_{\gamma} 0=0$, we have the existence of a $\gamma_{2} \in \Gamma$ such that $0 \notin$ $P_{\gamma} 0+U_{0} \quad\left(\gamma \geq \gamma_{2}\right)$. Finally, choose $\gamma_{0} \geq \gamma_{1}, \gamma_{2}$ and use, noting the circledness of $U_{0}$, the homotopie invariance of the Brouwer degree

2.3 Three fixed-point theorems. At the end of this section we transfer two fixedpoint theorems from Kaballo [6] to our situation and prove a theorem of Hahn and Pötter [4].

Theorem 7. Let $X$ be a topological vector space and $\left(P_{\gamma}\right)_{\gamma \in \Gamma \text { an approximation }}$ net in $X$. Suppose that every $P_{\gamma}$ maps bounded sets into bounded sets ${ }^{1)}$ and that assumption (iii) of Theorem 5 holds. Furthermore, let $\Omega$ be an open neighbourhood of zero and $F: \bar{\Omega} \rightarrow X$ a continuous map with bounded image. For every $t \in(0,1]$ suppose

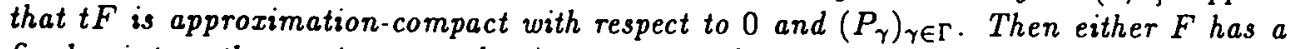
fixed point or there exist a $t_{0} \in[0,1)$ and a $x_{0} \in \partial \Omega$ with $x_{0}=t_{0} F x_{0}$.

Proof. If $F$ has a fixed point nothing is to do. Otherwise, because of the suppositions, $P_{\gamma} t F$ has bounded image for every $t \in[0,1)$ and $\gamma \in \Gamma$. Therefore, (4) holds for any $t F$ and we can use our degree concerning these maps, i.e. $\operatorname{deg}(I-t F, \Omega, 0)$ is defined for $t \in[0,1]$.

\footnotetext{
1). In case of a locally bounded space $X$ and an approximation sequence this assumption is redundant.
} 
Define the homotopy

$$
H(t, x)=t F_{x} \quad(x \in \bar{\Omega}, t \in[0,1]) .
$$

The assumptions (ii) and (iii) of Theorem 5 hold for $y(t) \equiv 0$. Assumption (i) of Theorem 5 follows from the bounded image of $F$ and so the homotopy invariance (G3) provides in conclusion with the normalization (G1)

$$
\operatorname{deg}(I-F, \Omega, 0)=\operatorname{deg}(I, \Omega, 0)=\{1\}
$$

noting that $\Omega$ is a neighbourhood of zero. Because of Theorem $3 /(i i)$, we have the existence of a fixed point and thus a contradiction

Theorem 8. Suppose the same assumptions as in Theorem 7. Suppose further that $\Omega$ is a shrinkable neighbourhood of zero and $F(\bar{\Omega}) \subseteq \bar{\Omega}$. Then $F$ has a fixed point.

Proof. If there exists a fixed point on $\partial \Omega$ we are done. In the other case we use the homotopy

$$
H(t, x)=t F x \quad(x \in \bar{\Omega}, t \in[0,1])
$$

again. Noting that $\Omega$ is shrinkable assumption (ii) of Theorem 5 holds and the rest of the proof is obvious

Theorem 9 (see Hahn and Pötter [4]). Let $\emptyset \neq \Omega \subseteq X$ be an open neighbourhood of zero and $F: \bar{\Omega} \rightarrow X$ be continuous and compact. Moreover, suppose that, for all $x \in \partial \Omega$ and $\alpha \in[0,1]$,

$$
F(x)=\alpha x \quad \text { implies } \quad \alpha \leq 1
$$

Then $F$ has a fixed point.

Proof. The maps $t F \quad(t \in[0,1])$ are approximation-compact with respect to 0 and the natural approximation net. If $F$ has a fixed point on $\partial \Omega$ we are done. In the other case we have to prove the requirements of Theorem 5 concerning the homotopy $H(t, x)=t F x \quad(x \in \bar{\Omega}, t \in[0,1])$ : Condition (i) follows from the compactness of $F$ and to prove condition (iii) we only have to look at the indices of the natural net. Finally, condition (ii) is provided by the assumed implication noting that $\alpha \neq 1$ is valid in the current case

The next section deals with the relationship between condensing maps and approximation-compact maps. It turns out that, if we consider the generalized degree as an extension of the degree for condensing maps, the results are not as natural as in case of compact maps. 


\section{Measures of non-compactness and $\psi$-condensing maps}

The purpose of a measure of non-compactness $r$ is the identification of relatively compact sets. In contrast to the definition of Sadovskii [15] at which the measure is invariant if we pass to the convex hull of a given set $A$, i.e. $r(A)=r(\operatorname{conv} A)$, we omit this part of the definition, because of our setting of a general topological linear space.

Deflnition 4. Let $X$ be a topological linear space and $\mathcal{M}$ a system of subsets of $X$ which includes all relatively compact sets. For a given set $A \neq \emptyset$ let $\mathcal{F}(A,[0, \infty])$ denote the system of all functions defined on $A$ with values in $[0, \infty]$. There is a natural partial ordering on $\mathcal{F}(A,[0, \infty])$ given by

$$
f \geq g \quad \Longleftrightarrow \quad f(a) \geq g(a) \text { for all } a \in A .
$$

A map $r: \mathcal{M} \rightarrow \mathcal{F}(A,[0, \infty])$ is called a measure of non-compactness if for all $M \in \mathcal{M}$ (R1) $r(M)=0$ if and only if $M$ relatively compact

(R2) $\bar{M} \in \mathcal{M}$ implies $r(M)=r(\bar{M})$.

If $A$ consists only of one element we identify $\mathcal{F}(A,[0, \infty])$ with $[0, \infty]$.

Example 1. The ball measure ${ }^{2)}$ of non-compactness is defined as follows: Let $A=\left(f_{\alpha}\right)_{\alpha \in \mathcal{A}}$ be a family of $F$-seminorms defining the topology of a complete topological linear space $X$. For every $\alpha \in \mathcal{A}$ let $B_{\alpha}(x, \vartheta) \quad(x \in X, \vartheta \in[0, \infty])$ denote the ball of radius $\vartheta$ around $x$ with respect to the semimetric induced by $f_{\alpha}$. In connection with this let us agree with $B_{\alpha}(x, \infty)=X$. We define for $M \in \mathcal{M}=\mathcal{P}(X)$

$$
r_{b}(M)\left(f_{\alpha}\right):=\inf \left\{\vartheta>0 \mid M \subseteq \bigcup_{m^{\prime} \in M^{\prime}} \overline{B_{\alpha}\left(m^{\prime}, \vartheta\right)} \text { for some finite } M^{\prime} \subseteq M\right\} .
$$

Proof. Clearly, we have property (R2), and property (R1) follows from the equivalence of relative compactness and precompactness in complete spaces

Furthermore, the above defined ball measure has the following two important properties of a measure of non-compactness:

Monotonicity, i.e. for all $M ; N \in \mathcal{M}, M \subseteq N$ implies $r(M) \leq r(N)$

Algebraic semi-additivity, i.e. for all $M, N \in \mathcal{M}, M+N \in \mathcal{M}$ implies $r(M+N) \leq$ $r(M)+r(N)$.

Example 2. Let $X$ be a complete $F$-normed linear space with $F$-norm $\|\cdot\|$ and $\left(P_{n}\right)_{n \in \mathbb{N}}$ an approximation sequence in $X$. Suppose that $P_{n}$ maps bounded sets into bounded sets. Let $\mathcal{M}$ denote the system of all bounded subsets of $X$. Then

$$
r_{p}(M)=\limsup \left(\sup _{x \in M}\left\|\left(I-P_{n}\right) x\right\|\right) \quad(M \in \mathcal{M})
$$

defines a monotonous measure of non-compactness.

2) Sadovskii [15] uses the term "Hausdorff measure" instead of "ball measure". 
Proof: Property (R2) follows from the continuity of $I, P_{n}$ and $\|\cdot\|$. The monotony is evident and the sufficiency of property (R1) holds, since $\left(P_{n}\right)_{n \in \mathbb{N}}$ is an approximation sequence.

To prove the necessity of property (R1) choose $M \in M$ with $r_{p}(M)=0$. We show that every sequence $\left(x_{m}\right)_{m \in \mathbb{N}}$ in $M$ has a Cauchy subsequence. Since $r_{p}$ is monotonous, we have

$$
r_{p}\left(\left\{x_{m} \mid m \in \mathbb{N}\right\}\right)=\lim \sup _{n}\left(\sup _{m \in \mathbb{N}}\left\|\left(I-P_{n}\right) x_{m}\right\|\right)=0 .
$$

The sets $\left\{P_{n} x_{m} \mid m \in \mathbb{N}\right\} \quad(n \in \mathbb{N})$ are relatively compact, noting that $P_{n}$ maps bounded sets into bounded sets and using the theorem of Heine-Borel. Therefore, we have inductively

$$
\begin{aligned}
n=1: & \left(P_{1} x_{m}\right)_{m \in \mathbb{N}} \text { has a Cauchy subsequence }\left(P_{1} x_{m_{k(1)}}\right)_{k \in \mathbb{N}} \\
n \rightarrow n+1: & \left(P_{n+1} x_{m_{k(n)}}\right)_{k \in \mathbb{N}} \text { has a Cauchy subsequence }\left(P_{n+1} x_{m_{k(n+1)}}\right)_{k \in \mathbb{N}} .
\end{aligned}
$$

For every $n \in \mathbb{N}$ we have

$$
\left(x_{m_{k(n+1)}}\right)_{k \in \mathbb{N}} \subseteq\left(x_{m_{k(n)}}\right)_{k \in \mathbb{N}}
$$

and it turns out that the diagonal sequence $\left(x_{m_{n(n)}}\right)_{n \in \mathbb{N}}$ is a Cauchy sequence. Indeed, fix $\varepsilon>0$. Because of (10) there exists an $n_{1} \in \mathbb{N}$ with

$$
\left\|\left(I-P_{n_{1}}\right) x_{m}\right\| \leq \frac{\varepsilon}{3} \quad(m \in \mathbb{N})
$$

Again, (10) provides the existence of an $n_{2} \in \mathbb{N}$ such that

$$
\left\|P_{n_{1}} x_{m_{n(n)}}-P_{n_{1}} x_{m_{n^{\prime}\left(n^{\prime}\right)}}\right\| \leq \frac{\varepsilon}{3} \quad\left(n, n^{\prime} \geq \max \left\{n_{1}, n_{2}\right\}\right)
$$

noting that $\left(P_{n_{1}} x_{m_{k\left(n_{1}\right)}}\right)_{k_{\in} \mathbb{N}}$ is a Cauchy sequence. Finally,

$$
\begin{aligned}
\left\|x_{m_{n(n)}}-x_{m_{n^{\prime}\left(n^{\prime}\right)}}\right\| \leq & \left\|x_{m_{n(n)}}-P_{n_{1}} x_{m_{n(n)}}\right\| \\
& +\left\|P_{n_{1}} x_{m_{n(n)}}-P_{n_{1}} x_{m_{n^{\prime}\left(n^{\prime}\right)}}\right\| \\
& +\left\|P_{n_{1}} x_{m_{n^{\prime}\left(n^{\prime}\right)}}-x_{m_{n^{\prime}\left(n^{\prime}\right)}}\right\| \\
\leq & \varepsilon
\end{aligned}
$$

shows that $\left(x_{m_{n(n)}}\right)_{n \in \mathbb{N}}$ is a Cauchy sequence and, together with $\left(x_{m_{n(n)}}\right)_{n \in \mathbb{N}} \subseteq$ $\left(x_{m}\right)_{m \in \mathbb{N}}$, the relative compactness of $M$ is shown

Now we introduce the notion of a $\psi$-condensing map which links approximationcompact maps with measures of non-compactness. The motivation of the following definition is due to Subsection 4.4 , but it can also be seen as a generalization of the notion of a condensing map defined by Sadovskii [15].3)

3) Sadovskii uses the character $\psi$ to name the measure of non-compactness. 
Definition 5. Let $\emptyset \neq \Omega \subseteq X$ be open and $r: \mathcal{M} \rightarrow \mathcal{F}(A,[0, \infty])$ be a measure of non-compactness with $M \cap \bar{\Omega} \in \mathcal{M}$ for every $M \in \mathcal{M}$. Suppose a map $F: \bar{\Omega} \rightarrow X$ with the property that $F(M \cap \bar{\Omega}) \in \mathcal{M}$ holds for every $M \in \mathcal{M}$. Let $\psi: \mathcal{F}(A,[0, \infty]) \rightarrow$ $\mathcal{F}(A,[0, \infty])$ be a monotonously increasing map with $\psi(f)=0$ if and only if $f=0$, for every $f \in \mathcal{F}(A,[0, \infty])$. Then $F$ will be called $\psi$-condensing with respect to the measure of non-compactness $r$ if the implication

$$
\psi(r(M \cap \bar{\Omega})) \leq r(F(M \cap \bar{\Omega})) \quad \Longrightarrow \quad M \cap \bar{\Omega} \text { is relatively compact }
$$

holds for every $M \in \mathcal{M}$.

If the measure of non-compactness is fixed let us agree to call such a map " $\psi$ condensing". In the same manner let us use " $k$-condensing" if $\psi=k I$, and "condensing" if $\psi=I$ which is in accordance with the definition of Sadovskii.

The following lemma links $\psi$-condensing mappings and approximation-compact mappings.

Lemma 2. Let $X$ be a Hausdorff linear space and $\left(P_{\gamma}\right)_{\gamma \in \Gamma \text { an approximation net }}$ in $X$. Suppose a measure of non-compactness $r: \mathcal{M} \rightarrow \mathcal{F}(A,[0, \infty])^{4)}$ and a function $\psi$ as in Definition 5. Let $\emptyset \neq \Omega \subseteq X$ be open and suppose a continuous map $F: \bar{\Omega} \rightarrow X$ which is $\psi$-condensing. Finally, assume the following estimates:

(i) There exists $\emptyset \neq Y \subseteq X$ such that, for all $y \in Y$ and $\Gamma^{\prime} \subseteq \Gamma$, if

$$
\lim _{\gamma^{\prime}}\left(x_{\gamma^{\prime}}-P_{\gamma^{\prime}} F x_{\gamma^{\prime}}\right)=y \quad \text { for some } x_{\gamma^{\prime}} \in \bar{\Omega}_{\gamma^{\prime}}
$$

then

$$
r\left(\left\{x_{\gamma} \mid \gamma^{\prime} \in \Gamma^{\prime}\right\}\right) \leq r\left(\left\{P_{\gamma^{\prime}} F x_{\gamma^{\prime}} \mid \gamma^{\prime} \in \Gamma^{\prime}\right\}\right) .
$$

(ii) For all $\Gamma^{\prime} \subseteq \Gamma$ and $\left(x_{\gamma^{\prime}}\right)_{\gamma^{\prime} \in \Gamma^{\prime}} \subseteq F(\Omega)$,

$$
\psi\left(r\left(\left\{P_{\gamma^{\prime}} x_{\gamma^{\prime}} \mid \gamma^{\prime} \in \Gamma^{\prime}\right\}\right)\right) \leq r\left(\left\{x_{\gamma^{\prime}} \mid \gamma^{\prime} \in \Gamma^{\prime}\right\}\right)
$$

Then $F$ is approximation-compact with respect to $Y$ and $\left(P_{\gamma}\right)_{\gamma \in \Gamma \text {. }}$.

Proof. Let $\left(x_{\gamma^{\prime}}\right)_{\gamma^{\prime} \in \Gamma^{\prime}}$ and $y \in Y$ as in assumption (i), i.e. $\left(x_{\gamma^{\prime}}\right)_{\gamma^{\prime} \in \Gamma^{\prime}}$ fulfils assumption (A). Linking assumptions (i) and (ii) and the monotony of $\psi$ we have

$$
\psi\left(r\left(\left\{x_{\gamma^{\prime}} \mid \gamma^{\prime} \in \Gamma^{\prime}\right\}\right)\right) \leq r\left(\left\{F x_{\gamma^{\prime}} \mid \gamma^{\prime} \in \Gamma^{\prime}\right\}\right)
$$

which implies the relative compactness of $\left\{x_{\gamma^{\prime}} \mid \gamma^{\prime} \in \Gamma^{\prime}\right\}$. There exists a subnet $\Gamma^{\prime \prime}$ of $\Gamma^{\prime}$ and an element $x \in \bar{\Omega}$ such that $\lim _{\gamma^{\prime \prime}} x_{\gamma^{\prime \prime}}=x$. Since $\left\{x_{\gamma^{\prime \prime}} \mid \gamma^{\prime \prime} \in \Gamma^{\prime \prime}\right\}$ is relatively compact and $F$ is continuous we obtain $x-F x=y$ noting that $\left(P_{\gamma^{\prime \prime}}\right)_{\gamma^{\prime \prime} \Gamma^{\prime \prime}}$ is an approximation net

4) The domain $\mathcal{M}$ of the measure is not specified. At the beginnig of each application of the lemma we have to control that all sets used as arguments for $r$ are contained in $\mathcal{M}$. 
Estimate (i) of the above Lemma 2 is a technical one. E.g., in case of an algebraically semi-additive measure of non-compactness it holds for approximation sequences. For the measure $r_{p}$ we obtain next a concrete function $\psi$.

Corollary 1. Let $X$ be a complete $F$-normed space with $F$-norm $\|\cdot\|$ and $\left(P_{n}\right)_{n \in \mathbb{N}}$ an approximation sequence in $X$. Let $\emptyset \neq \Omega \subseteq X$ be an open set and $F: \bar{\Omega} \rightarrow X$ a continuous map with bounded image. Furthermore, suppose the following: defined).

(i) For every bounded set $M \subseteq X, \cup_{n \in \mathbb{N}} P_{n}(M)$ is bounded (especially, $r_{p}$ is

(ii) $\left\{P_{n}\right\}_{n \in \mathbb{N}}$ is uniformly equicontinuous on bounded sets.

(iii) For all $n, m \in \mathbb{N}, X_{n} \subseteq X_{n+1},\left.P_{n}\right|_{X_{n}}=I$ and $P_{m} \circ P_{n}=P_{\min \{m, n\}}$.

Then estimates (i) and (ii) of Lemma 2 hold for $Y=\{0\}$ and $\psi=\frac{1}{2} I$.

Therefore, using Lemma 2, every continuous map with bounded image which is $\frac{1}{2}$ condensing with respect to $r_{p}$ is approximation-compact with respect to 0 and $\left(P_{n}\right)_{n \in \mathbb{N}}$.

Proof. We have to prove estimates (i) and (ii) of Lemma 2. Let $\left(x_{n_{k}}\right)_{k \in \mathbb{N}}$ with $x_{n_{k}} \in \bar{\Omega}_{n_{k}}$ for $k \in \mathbb{N}$ and

$$
\lim _{k}\left(x_{n_{k}}-P_{n_{k}} F x_{n_{k}}\right)=0
$$

as given in estimate (i) of Lemma 2 with $y=0$. The set $\left\{F x_{n_{k}} \mid k \in \mathbb{N}\right\}$ is bounded since $F(\bar{\Omega})$ is bounded. Linking (i) and (11) we obtain that $\left\{x_{n_{k}} \mid k \in \mathbb{N}\right\}$ is bounded, too. Now $r_{p}\left(\left\{x_{n_{k}} \mid k \in \mathbb{N}\right\}\right), r_{p}\left(\left\{F x_{n_{k}} \mid k \in \mathbb{N}\right\}\right), r_{p}\left(\left\{P_{n_{k}} F x_{n_{k}} \mid k \in \mathbb{N}\right\}\right)$ are defined. We have the equalities

$$
\begin{aligned}
r_{p}\left(\left\{x_{n_{k}} \mid k \in \mathbb{N}\right\}\right) & =\underset{n}{\limsup }\left(\sup _{k \in N}\left\|\left(I-P_{n}\right) x_{n_{k}}\right\|\right) \\
& =\underset{n}{\limsup }\left(\sup _{n_{k}>n}\left\|\left(I-P_{n}\right) x_{n_{k}}\right\|\right)
\end{aligned}
$$

because of the retract condition (iii). Since the family $\left\{I-P_{n}\right\}_{n \in \mathbb{N}}$ is equicontinuous in zero and using (11) and (12) we have

$$
\begin{aligned}
r_{p}\left(\left\{x_{n_{k}} \mid k \in \mathbb{N}\right\}\right) & =\underset{n}{\limsup }\left(\sup _{n_{k}>n}\left\|\left(I-P_{n}\right)\left(x_{n_{k}}-\left(x_{n_{k}}-P_{n_{k}} F x_{n_{k}}\right)\right)\right\|\right) \\
& =\underset{n}{\limsup }\left(\sup _{n_{k}>n}\left\|\left(I-P_{n}\right) P_{n_{k}} F x_{n_{k}}\right\|\right) \\
& =r_{p}\left(\left\{P_{n_{k}} F x_{n_{k}} \mid k \in \mathbb{N}\right\}\right)
\end{aligned}
$$

and estimate (i) of Lemma 2 is shown.

To prove estimate (ii) of Lemma 2 we can work with sequences $\left(x_{k}\right)_{k \in \mathbb{N}}$ instead of the subsequences used in Lemma 2 noting that $\psi$ and $r_{p}$ are monotonous. That 
$r_{p}\left(\left\{x_{k} \mid k \in \mathbb{N}\right\}\right)$ and $r_{p}\left(\left\{P_{k} x_{k} \mid k \in \mathbb{N}\right\}\right)$ are defined can be shown in the same way as before. Using assumption (iii) we obtain

$$
\begin{aligned}
\frac{1}{2} r_{p}\left(\left\{P_{k} x_{k} \mid k \in \mathbb{N}\right\}\right) & =\frac{1}{2} \limsup _{n}\left(\sup _{k \in \mathbb{N}}\left\|\left(I-P_{n}\right)\left(P_{k} x_{k}\right)\right\|\right) \\
& =\frac{1}{2} \limsup _{n}\left(\sup _{k \in \mathbb{N}}\left\|P_{k} x_{k}-P_{n} P_{k} x_{k}\right\|\right) \\
& =\frac{1}{2} \limsup _{n}\left(\sup _{k>n}\left\|P_{k} x_{k}-P_{n} x_{k}\right\|\right)
\end{aligned}
$$

followed by the estimate

$$
\begin{aligned}
& \frac{1}{2} \limsup _{n}\left(\sup _{k>n}\left\|P_{k} x_{k}-P_{n} x_{k}\right\|\right) \\
& \quad \leq \frac{1}{2} \limsup _{n}\left(\sup _{k>n}\left(\left\|P_{k} x_{k}-x_{k}\right\|+\left\|x_{k}-P_{n} x_{k}\right\|\right)\right) \\
& \leq \frac{1}{2} \lim _{n}\left(\sup _{l \geq n}\left(\sup _{k \geq l}\left\|\left(I-P_{k}\right) x_{k}\right\|+\frac{1}{2} r_{p}\left(\left\{x_{k} \mid k \in \mathbb{N}\right\}\right)\right)\right) \\
& \leq \frac{1}{2} \lim _{n}\left(\sup _{l \geq n}\left(\sup _{k \in \mathbb{N}}\left\|\left(I-P_{l}\right) x_{k}\right\|+\frac{1}{2} r_{p}\left(\left\{x_{k} \mid k \in \mathbb{N}\right\}\right)\right)\right) \\
& \quad=r_{p}\left(\left\{x_{k} \mid k \in \mathbb{N}\right\}\right) .
\end{aligned}
$$

I.e., estimate (ii) of Lemma 2 is shown for $\psi=\frac{1}{2} I$

We refer to Subsection 4.4 for non-trivial examples of approximation sequences which hold properties (i) - (iii) of Corollary 1.

Our next section gives some examples of approximation nets that differ from the natural net. They all are sequences. The consequence is that the condition $x-F x=y$ of assumption (A) is redundant, since convergent sequences are relative compact. The bad effect of differing from the natural net is that one has to prove property (4) before using the generalized degree.

\section{Examples of approximation sequences}

4.1 General linear approximation sequences. In the foregoing section a lot of assumptions have been made concerning approximation nets and approximation sequences. If these approximations are linear the Banach-Steinhaus theorem is very useful.

In case of a linear approximation sequence $\left(P_{n}\right)_{n \in N}$ in a complete $F$-normed space $X$ the orbits $\left\{P_{n} x \mid n \in \mathbb{N}\right\}$ are bounded for every $x \in X$. Therefore, we have by the Banach-Steinhaus theorem 
Corollary 2. Let $X$ be a complete $F$-normed space and $\left(P_{n}\right)_{n \in \mathbb{N}}$ a linear approximation sequence in $X$. Then $\left\{P_{n}\right\}_{n \in \mathbb{N}}$ is an equicontinuous family.

If $X$ is locally bounded, the $F$-norm is given by a $p$-norm $\|\cdot\|_{p}$. I.e., there exists a $0<p \leq 1$ such that $\|\lambda x\|_{p}=|\lambda|^{p}\|x\| \quad(\lambda \in \mathbb{R}, x \in X)$ holds in addition to the properties of the $F$-norm. In this case a continuous linear operator $T: X \rightarrow X$ has a norm

$$
\|T\|=\sup \left\{\|T x\|_{p}^{1 / p} \mid\|x\|_{p}^{1 / p}=1\right\} \in \mathbb{R}_{0}^{+} .
$$

Corollary 3. Let $X$ be a complete p-normed space with p-norm $\|\cdot\|_{p}$ and $\left(P_{n}\right)_{n \in \mathbb{N}}$ a linear approximation sequence in $X$. Then $\sup _{n}\left\|P_{n}\right\|<\infty$.

Now, choosing $r_{b}$ as measure of non-compactness, we have an estimate for the weight function $\psi$ in Lemma 2.

Ccorollary 4. Suppose a complete p-normed space $X$ with p-norm $\|\cdot\|_{p}$ and a linear approximation sequence $\left(P_{n}\right)_{n \in \mathbb{N}}$ in $X$. Let $C:=\sup _{n}\left\|P_{n}\right\|$ and $\emptyset \neq \Omega \subseteq X$ be open. Then every continuous map $F: \bar{\Omega} \rightarrow X$ which is $C^{-p}$-condensing with respect to $r_{b}$ is approximation-compact.

Proof. Again, we prove the estimates (i) and (ii) of Lemma 2 with $\psi=t \mapsto C^{-p_{t}}$ and $Y=X$.

Estimate (i) follows from the algebraical subadditivity of $r_{b}$ noting that convergent sequences are relatively compact.

Concerning estimate (ii) we can work with sequences instead of arbitrary subsequences (see the proof of Corollary 1). Therefore, choose $\left(x_{n}\right)_{n \in \mathbb{N}} \subseteq X$ with $r_{b}\left(\left\{x_{n} \mid n \in\right.\right.$ $\mathbb{N}\})=\vartheta \in(0, \infty)$. For $\delta>0$ we have a finite subset $M_{\delta} \subseteq\left\{x_{n} \mid n \in \mathbb{N}\right\}$ such that $\left\|x_{n}-m_{n}\right\|_{p} \leq \vartheta+\delta(n \in \mathbb{N})$ for suitable chosen $m_{n} \in M_{\delta}$. Let $B_{p}(0, \alpha)$ denote the ball around zero of radius $\alpha$ with respect to the metric which is induced by the $p$-norm. The estimate

$$
\begin{aligned}
r_{b}\left(\left\{P_{n} x_{n} \mid n \in \mathbb{N}\right\}\right) & \leq r_{b}\left(\left\{P_{n} x_{n}-P_{n} m_{n} \mid n \in \mathbb{N}\right\}\right) \\
& =r_{b}\left(\left\{P_{n}\left(x_{n}-m_{n}\right) \mid n \in \mathbb{N}\right\}\right) \\
& \leq r_{b}\left(\cup_{n} P_{n}\left(\overline{B_{p}(0, \vartheta+\delta)}\right)\right) \\
& \leq \sup _{n}\left\|P_{n}\right\|^{p}(\vartheta+\delta) \\
& =C^{p}(\vartheta+\delta)
\end{aligned}
$$

concludes the proof by $\delta \rightarrow 0$

4.2 The sequence spaces $l^{p}$ for $0<p<1$. Let $x=\left(x_{n}\right)_{n \in \mathbb{N}} \subseteq \mathbb{R}$ and $0<p<1$. We define

$$
\|x\|_{p}=\sum_{i=1}^{\infty}\left|x_{i}\right|^{p} .
$$


This defines a $p$-norm on the sequence space

$$
l^{p}=\left\{\left(x_{n}\right)_{n \in \mathbb{N}} \subseteq \mathbb{R} \mid\|x\|_{p}<\infty\right\} .
$$

The induced metric defines a complete space which is not locally convex - see Landsberg [9]. However, $l^{p}$ is admissible and we obtain an approximation sequence as follows:

For every $i \in \mathbb{N}$ let $e_{i}:=\left(\delta_{i n}\right)_{n \in \mathbb{N}}$. The set $\left\{e_{i}\right\}_{i \in \mathbb{N}}$ is a Schauder base for $l^{p}$. Let $x=\left(x_{n}\right)_{n \in \mathbb{N}} \in l^{p}$. The mappings

$$
P_{m}(x)=\sum_{i=1}^{m} x_{i} e_{i} \quad(m \in \mathbb{I N})
$$

are continuous linear projections of norm 1 with finite-dimensional image. Therefore, they are uniformly equicontinuous on $l^{p}$. Using this and (13) we have that $\left(P_{n}\right)_{n \in \mathbb{N}}$ is an approximation sequence in $l^{p}$.

Let $\emptyset \neq \Omega \subseteq l^{p}$ be open. In case of the space $l^{p}$ the constant $C$ of Corollary 4 is equal to 1 . Therefore, every continuous map $F: \bar{\Omega} \rightarrow X$ which condenses with respect to $r_{b}$ is approximation-compact with respect to $\left(P_{n}\right)_{n \in \mathbb{N}}$.

Concerning the measure of non-compactness $r_{p}$ a short look at the proof of Corollary 1 and (13) shows that the corollary holds for maps which condense with respect to $r_{p}$ instead of the $\frac{1}{2}$-condensing ones.

Now we have by Theorem 8 the following

Theorem 10. Let $\emptyset \neq \Omega \subseteq l^{p}$ be an open and shrinkable neighbourhood of zero. Suppose a continuous map $F: \bar{\Omega} \rightarrow \bar{\Omega}$ with bounded image which condenses with respect to $r_{b}$ or $r_{p}$. Then $F$ has a fixed point.

Proof. First we notice that the generalized degree is defined. For example this follows from the boundedness of the image of $F$ linked with the linearity of the approximation sequence $\left(P_{n}\right)_{n \in \mathbb{N}}$. It is now easy to see that most of the assumptions of Theorem 8 hold. For example, $t F$ is approximation-compact, since $t F$ condenses for every $t \in(0,1)$ with respect to $r_{b}$ or $r_{p}$, respectively

Now we give an application of the above theorem by constructing a map $F: l^{p} \rightarrow l^{p}$ which condenses with respect to $r_{b}$.

Example 3. Let $\left(\varphi_{k}\right)_{k \in \mathbb{N}}$ be a family of equicontinuous maps $\varphi_{k}: \mathbb{R} \rightarrow \mathbb{R}$. Moreover, suppose the existence of a constant $M \in \mathbb{R}$ such that

$$
\sup _{k \in \mathbb{N}}\left\|\varphi_{k}\right\|_{\infty} \leq M
$$

Finally, let $a=\left(a_{i}\right)_{i \in \mathbb{N}}, b=\left(b_{i}\right)_{i \in \mathbb{N}} \in l^{p}$ and $\lambda \in \mathbb{R}$.

The problem to solve is an infinite-dimensional system of nonlinear equations

$$
x_{i}=\sum_{k=1}^{\infty} a_{i} 2^{-|k-i|} \varphi_{k}\left(x_{k}\right)+\lambda x_{i+1}+b_{i} \quad(i \in \mathbb{N}) .
$$


We will get a solution of system (14) in $l^{p}$ by making assumptions on $a=\left(a_{i}\right)_{i \in \mathbb{N}}, b=$ $\left(b_{i}\right)_{i \in \mathbb{N}}$ and $\lambda$ to use Theorem 10 with a suitable condensing map $F$ we define as follows.

Let $F_{1}, F_{2}: B_{p}(0, r) \rightarrow l^{p}$ be defined by

$$
\begin{aligned}
& \left(F_{1}(x)\right)_{i}=\sum_{k=1}^{\infty} a_{i} 2^{-|k-i|} \varphi_{k}\left(x_{k}\right) \\
& \left(F_{2}(x)\right)_{i}=\lambda x_{i+1}+b_{i}
\end{aligned}
$$

for every $x=\left(x_{i}\right)_{i \in \mathbb{N}} \in B_{p}(0, r)$. The estimate

$$
\left\|F_{2}(x)-F_{2}(y)\right\|_{p} \leq|\lambda|^{p}\|x-y\|_{p} \quad\left(x, y \in B_{p}(0, r)\right)
$$

shows that $F_{2}$ is defined, continuous, and $|\lambda|^{p}$-condensing with respect to $r_{b}$.

Concerning $F_{1}$ we first supplement the approximation sequence $\left(P_{n}\right)_{n \in \mathbb{N}}$ by setting $P_{n}=0$ for $n=0$. Let $x=\left(x_{k}\right)_{k \in \mathbb{N}} \in l^{p}$ and $n \in \mathbb{N}_{0}$. The case $n=0$ in the calculation

$$
\begin{aligned}
\left\|\left(I-P_{n}\right) F_{1}(x)\right\|_{p} & =\sum_{i=n+1}^{\infty}\left|\left(F_{1}(x)\right)_{i}\right|^{p} \\
& =\sum_{i=n+1}^{\infty}\left|\sum_{k=1}^{\infty} a_{i} 2^{-|k-i|} \varphi_{k}\left(x_{k}\right)\right|^{p} \\
& \leq \sum_{i=n+1}^{\infty}\left|a_{i}\right|^{p}\left(3-2^{-i+1}\right)^{p} M^{p}
\end{aligned}
$$

provides the estimate

$$
\left\|F_{1}(x)\right\|_{p} \leq 3^{p}\|a\|_{p} M^{p} \quad\left(x \in B_{p}(0, r)\right)
$$

and, therefore, $F_{1}$ is defined. The equicontinuity of the family $\left(\varphi_{k}\right)_{k \in \mathbb{N}}$ linked with the equation

$$
\left\|F_{1}(x)-F_{1}(y)\right\|_{p}=\sum_{i=1}^{\infty}\left|\sum_{k=1}^{\infty} a_{i} 2^{-|k-i|}\left(\varphi_{k}\left(x_{k}\right)-\varphi_{k}\left(y_{k}\right)\right)\right|^{p}
$$

which can be continued by an estimate like (15) provides the continuity of $F_{1}$. To show the compactness of $F_{1}$ we refer to the measure of non-compactness $r_{p}$ and (15). In fact, we have

$$
r_{p}\left(F_{1}(B(0, r))\right)=\underset{n}{\limsup }\left(\sup _{x \in B_{p}(0, r)}\left\|\left(I-P_{n}\right) F_{1}(x)\right\|_{p}\right)=0 .
$$

The map $F=F_{1}+F_{2}$ is continuous and condenses with respect to the measure of non-compactness $r_{b}$ for $|\lambda|<1$, since $r_{b}$ is algebraically semi-additive. The ball $B_{p}(0, r)$ is a bounded and shrinkable neighbourhood of zero and, finally, we have requirements on $M, \lambda, r, a=\left(a_{i}\right)_{i \in \mathbb{N}}$ and $b=\left(b_{i}\right)_{i \in \mathbb{N}}$ in form of an estimate which links them and provides that the image of $F$ is contained $B_{p}(0, r)$. Exactly, let $x \in B_{p}(0, r)$. Then

$$
\left\|F_{2}(x)\right\|_{p} \leq|\lambda|^{p}\|x\|_{p}+\|b\|_{p}
$$


linked with the estimate (16) provides

$$
\|F(x)\|_{p} \leq 3^{p}\|a\|_{p} M^{p}+|\lambda|^{p}\|x\|_{p}+\|b\|_{p} .
$$

If we assume

$$
\left(|\lambda|^{p}-1\right) r+3^{p}\|a\|_{p} M^{p}+\|b\|_{p} \leq 0
$$

which is the announced connection of the parameters the desired estimate for the image of $F$ follows. Therefore, (17) and $|\lambda|<1$ provide a solution of the fixed-point equation $F(x)=x$ in $B(0, r)$ which is equivalent to a solution $x$ of the system (14) in $l^{p}$ with $\|x\|_{p} \leq r$.

4.3 The Hardy spaces $H^{p}$ for $0<p<1$ and the Nevanlinna class $N^{+}$. Let $\mathbb{D}=\{z \in \mathbb{C}|| z \mid<1\}$ be the open unit disc in the complex plane and $H(D)$ be the set of all functions which are analytic on $\mathbb{D}$. Let $0 \leq r<1$ and define $T_{r}: H(\mathbb{D}) \rightarrow H\left(\frac{1}{r} \mathbb{D}\right)$ by

$$
T_{r}(f)(z)=f(r z) \quad\left(f \in H(\mathbb{D}), z \in \frac{1}{r} \mathbb{D}\right)
$$

with the agreement $\frac{1}{0} \mathbb{D}=\mathbb{C}$. Furthermore, for $f \in H(\mathbb{D})$, we define in the case $0<p<1$

$$
\|f\|_{p}=\sup _{0<r<1} \frac{1}{2 \pi} \int_{-\pi}^{+\pi}\left|\left(T_{r} f\right)\left(e^{i \theta}\right)\right|^{p} d \theta
$$

and

$$
\|f\|_{0}=\sup _{0<r<1} \frac{1}{2 \pi} \int_{-\pi}^{+\pi} \log _{+}\left|\left(T_{r} f\right)\left(e^{i \theta}\right)\right| d \theta .
$$

for $p=0$.

We obtain the Hardy spaces $H^{P}$ and the Nevanlinna class $N$ by

$$
H^{p}=\left\{f \in H(\mathbb{D}) \mid\|f\|_{p}<\infty\right\} \quad \text { and } \quad N=\left\{f \in H(\mathbb{D})\|\| f \|_{0}<\infty\right\}
$$

respectively.

For every $f \in N$ the non-tangential limits $\lim _{r \rightarrow 1-1}\left(T_{r} f\right)\left(e^{i \theta}\right)$ exist a.e. and we have an identification with a measurable function on $\partial I D$ which will be denoted by $f$, too (see Duren [3]). In case of $f \in H^{p}$ the identification provides

$$
\|f\|_{p}=\frac{1}{2 \pi} \int_{-\pi}^{+\pi} \mid f\left(\left.e^{i \theta}\right|^{p} d \theta\right.
$$

For general $f \in N$ this property characterises a subclass $N^{+}$of $N$ :

$$
N^{+}=\left\{\begin{array}{l|l}
\left.f \in N\left|\|f\|_{0}=\frac{1}{2 \pi} \int_{-\pi}^{+\pi} \log _{+}\right| f\left(e^{i \theta}\right) \mid d \theta\right\}
\end{array}\right.
$$


and we have the inclusions $H^{q} \subseteq H^{p} \subseteq N^{+} \subseteq N$ for $0<p<q<1$.

The norms $\|\cdot\|_{p}$ are $p$-norms for the spaces $H^{p} \quad(0<p<1)$. These complete spaces are not locally convex (see Landsberg [9]). The space $N$ can be provided with an $F$-norm

$$
\|f\|_{N}=\frac{1}{2 \pi} \int_{-\pi}^{+\pi} \log \left(1+\left|f\left(e^{i \theta}\right)\right|\right) d \theta \quad(f \in N) .
$$

The obtained metric space is not locally bounded and only the subclass $N^{+}$is complete with respect to this metric (see Yanagihara [19]).

To show the admissibility of $H^{p}$ and $N^{+}$by defining approximation sequences we use the proof of the admissibility which has been given by Krauthausen [8]. The operators $\left.T_{r}\right|_{H^{\circ}}$ and $\left.T_{r}\right|_{N^{+}}$are Lipschitz continuous with Lipschitz constant 1 (see Rudin [14] and Yanagihara [19]). It is easy to see that for given $\varepsilon>0$ and compact $K \subseteq H^{p}$ or $K \subseteq N^{+}$we have an $r \in[0,1)$ such that

$$
\left\|T_{r} f-f\right\|_{p} \leq \frac{\varepsilon}{2} \quad \text { or } \quad\left\|T_{r} f-f\right\|_{N} \leq \frac{\varepsilon}{2}
$$

holds for every $f \in K$, respectively.

In a second step we approximate $g \in T_{r}(K) \subseteq H(\mathbb{D})$ on $\partial \mathbb{D}$ by using Taylor polynomials: Let $r \in[0,1)$ and $f \in H\left(\frac{1}{r} \mathbb{D}\right)$ be fixed. Let $M_{f}(\delta)$ denote the maximum of $|f|$ on $\partial(\delta D)$ for $\delta \in\left[0, \frac{1}{r}\right)$. For $f \in H^{p}$ and $f \in N^{+}$we have (see Priwalow [11]) the estimates

$$
M_{f}(\delta) \leq\left(\frac{2\|f\|_{p}}{1-\delta}\right)^{1 / p} \quad \text { and } \quad M_{f}(\delta) \leq e^{2\|f\|_{0} /(1-\delta)} \quad(0 \leq \delta<1)
$$

respectively. Varying $f$ in $K$ we provide

$$
C_{K, r}:=\sup _{f \in K} M_{T_{r} f}(1)<\infty
$$

noting that $\|\cdot\|_{0} \leq\|\cdot\|_{N}$. For every $n \in N_{0}$ let

$$
P_{n}(f)(z):=\sum_{k=0}^{n} a_{k}(f) z^{k} \quad(|z|<1)
$$

denote the $n$-th Taylor polynomial of $f \in H(D)$. For short, let $r^{\prime}:=\frac{1}{2}\left(\frac{1}{r}+1\right)$ and $r^{\prime \prime}:=\frac{1}{2}(r+1) \quad(r \in(0,1])$. By the Cauchy formula and (21) we have

$$
\begin{aligned}
M_{\left(I-P_{n}\right) T_{r} f}(1) & \leq \sum_{k=n+1}^{\infty}\left|a_{k}\right|\left(T_{r} f\right) \\
& \leq \sum_{k=n+1}^{\infty} M_{T_{r}, f}(1) r^{\prime-k} \quad(f \in K) \\
& \leq C_{K, r^{\prime \prime}} \sum_{k=n+1}^{\infty} r^{\prime-k} .
\end{aligned}
$$


Choosing $n=n(K, r)$ such that the last term can be estimated by $\min \left\{\frac{\varepsilon}{2}{ }^{1 / p}, e^{\varepsilon / 2}-1\right\}$ the terms $\left\|\left(I-P_{n}\right) T_{r} f\right\|_{p}$ and $\left\|\left(I-P_{n}\right) T_{r} f\right\|_{N}$ can be estimated by $\frac{\varepsilon}{2}$ independent of $f \in K$. Linked with our first $\frac{e}{2}$-argument the admissibility of $H^{p}$ and $N^{+}$follows directly from the triangle inequality.

To define an approximation sequence we have to link $n$ and $r$. For short, let $r_{n}:=$ $\left(1-\frac{1}{n}\right) \quad(n \in \mathbb{N})$. Let $\left(m_{n}\right)_{n \in \mathbb{N}}$ denote a monotonous increasing sequence of natural numbers we specify further on. In case of the Hardy spaces we have

$$
\sup _{f \in K} M_{\left(I-P_{m_{n}}\right) T_{r_{n}} f}(1) \leq \sup _{f \in K}\left(4 n\|f\|_{p}\right)^{1 / p} \sum_{k=m_{n}+1}^{\infty}\left(\frac{2 n-2}{2 n-1}\right)^{k}
$$

referring to (20) - (22). Now choose $m_{n} \in \mathbb{N}$ such that

$$
(4 n)^{1 / p} \sum_{k=m_{n}+1}^{\infty}\left(\frac{2 n-2}{2 n-1}\right)^{k}<\frac{1}{n} \text {. }
$$

Then, defining $P_{n}^{*}=P_{m_{n}} \circ T_{r_{n}}(n \in \mathbb{N})$, the sequence of mappings $\left(P_{n}^{*}\right)_{n \in \mathbb{N}}$ defines a linear approximation sequence.

Finally, we obtain an analogous result for the Nevanlinna class $N^{+}$. Starting with a sequence $\left(l_{n}\right)_{n \in \mathbb{N}}$ instead of $\left(m_{n}\right)_{n \in \mathbb{N}}$ we have the estimate

$$
M_{\left(I-P_{l_{n}}\right) T_{r_{n}} f}(1) \leq \sup _{f \in K} e^{4 n\|f\|_{N}} \sum_{k=l_{n}+1}^{\infty}\left(\frac{2 n-2}{2 n-1}\right)^{k}
$$

Choose $\left(l_{n}\right)_{n \in \mathbb{N}}$ such that

$$
n^{n} \sum_{k=I_{n}+1}^{\infty}\left(\frac{2 n-1}{2 n-2}\right)^{k}<\frac{1}{n} \quad(n \in \mathbb{N})
$$

The mappings

$$
P_{n}^{*}:=P_{l_{n}} \circ T_{r_{n}}
$$

define a linear approximation sequence in $N^{+}$and every $H^{p}$ since for every $p \in(0,1)$ we have an $n_{p} \in \mathbb{N}$ with $n^{n} \geq(4 n)^{1 / p}$ for all $n \geq n_{p}$.

We look for fixed-point theorems for $\psi$-condensing maps as we have done in case of the sequence spaces $l^{p}$. Our first available lemma which links approximation-compact maps with condensing maps, Corollary 1, does not work, since it's retract assumptions do not hold.

Concerning the measure $r_{b}$ we have to use Corollary 4. In case of the Hardy spaces it turns out a statement concerning $C^{-p}$-condensing maps with a constant $C>0$ which we do not know. In case of the Nevanlinna class $\mathrm{N}^{+}$we obtain no result.

To "solve" this problem we introduce $F$-norms in $H^{p}$ and $N^{+}$which harmonize with the approximations:

$$
\|f\|_{p}^{\prime}=\sup _{n \in \mathbb{N}}\left\|P_{n}^{*} f\right\|_{p} \quad \text { and } \quad\|f\|_{N}^{\prime}=\sup _{n \in \mathbb{N}}\left\|P_{n}^{*} f\right\|_{N}
$$


for $f \in H^{p}$ and $f \in N^{+}$, respectively. The reader will convince himself that $\|\cdot\|_{p}^{\prime}$ is a p-norm and $\|\cdot\|_{N}^{\prime}$ is an $F$-norm. To show that $\|\cdot\|_{p}^{\prime}$ and $\|\cdot\|_{N}^{\prime}$ induces the same notion of convergence as $\|\cdot\|_{P}$ and $\|\cdot\|_{N}$ does, respectively, we calculate, for example,

$$
\begin{aligned}
\left\|P_{n}^{*} f\right\|_{p}^{\prime} & =\sup _{m \in \mathbb{N}}\left\|P_{m}^{*} P_{n}^{*} f\right\|_{p}=\sup _{m \in \mathbb{N}}\left\|P_{l_{m}} P_{l_{n}} T_{r_{m}} T_{r_{n}} f\right\|_{p} \\
& =\sup _{\substack{m \in \mathbb{N} \\
k=\min (m, n)}}\left\|P_{l_{k}} T_{r_{m} r_{n}} f\right\|_{p} \leq \sup _{k \in \mathbb{N}}\left\|P_{l_{k}} \dot{T}_{r_{k}} f\right\|_{p} \leq\|f\|_{p}^{\prime},
\end{aligned}
$$

noting that the first estimate holds, since $P_{l_{k}}$ is subharmonic. We obtain the estimates $\left\|P_{n}^{*}\right\| \leq 1 \quad(n \in \mathbb{N})$. Since $P_{n}^{*}(f)=f$ for constant $f$ we have $\left\|P_{n}^{*}\right\|=1 \quad(n \in \mathbb{N})$ with respect to $\|\cdot\|_{p}^{\prime}$ in case of the Hardy spaces. In case of the Nevanlinna class we have 1 as a Lipschitz constant for the approximations $P_{n}^{*}$ using the same argumentation as above.

The space $H^{p}$ is complete with respect to the new $F$-norm and using Corollary 4 we have: that for $\emptyset \neq \Omega \subseteq H^{p}$ every continuous map $F: \bar{\Omega} \rightarrow H^{p}$ which condenses with respect to $r_{b}^{\prime}$ is approximation-compact, at which $r_{b}^{\prime}$ denotes the ball measure of non-compactness with respect to the new $F$-norm. The same statement holds for the Nevanlinna class $N^{+}$noting that the constant $C^{-p}$ of Corollary. 4 is used as a Lipschitz constant only.

Theorem 11. Let $\emptyset \neq \Omega \subseteq H^{P}$ or $\emptyset \neq \Omega \subseteq N^{+}$be an open shrinkable neighbour. hood of zero. Suppose a continuous map $F: \bar{\Omega} \rightarrow \bar{\Omega}$ which has bounded image and condenses with respect to $r_{b}^{\prime}$. Then $F$ has a fixed point.

Proof. In case of the Hardy spaces the proof of Theorem 10 can be adopted. Concerning the Nevanlinna class we first notice that we can use the generalized degree since $P_{n}^{*}$ is linear for $n \in \mathbb{N}$ and the image of $F$ is bounded. Again, it is easy to see that most of the assumptions of Theorem 8 hold. We only show that $t F$ condenses with respect to $r_{b}^{\prime}$ for every $t \in(0,1)$.

Let $B_{N^{\prime}}(m, \vartheta)$ denote the ball of radius $\vartheta>0$ around $m$ with respect to the metric induced by $\|\cdot\|_{N}^{\prime}$. Choose $\hat{x} \in N^{+}$and $t \in(0,1)$. The calculation

$$
\begin{aligned}
\hat{x} \in t B_{N^{\prime}}(m, \vartheta) & \Longleftrightarrow \quad \hat{x} \in\left\{t x \mid\|x-m\|_{N}^{\prime}<\vartheta\right\} \\
& \Longleftrightarrow \quad \hat{x} \in\left\{y \mid\left\|\frac{y}{t}-m\right\|_{N}^{\prime}<\vartheta\right\} \\
& \Longleftrightarrow \quad \hat{x} \in\left\{y \mid\|y-t m\|_{N}^{\prime}<\vartheta\right\} \\
& \Longleftrightarrow \quad \hat{x} \in B_{N^{\prime}}(t m, \vartheta) .
\end{aligned}
$$

is all we need

Again, as an application of the fixed-point theorem we analyze an operator concerning his fixed points.

Example 4. Suppose $p=\frac{1}{2}$ is fixed. As in Example 3 the operator $F$ is a linear contraction $F_{\lambda}$ perturbed by a nonlinear compact operator $F_{\varphi}$. Choosing $\lambda \in[0,1)$ every continuous linear operator $F_{\lambda}: H^{1 / 2} \rightarrow H^{1 / 2}$ with norm $\lambda$ condenses with respect to $r_{b}$. 
The definition of the compact perturbation is more complicated: Define $S: H(\mathbb{D}) \rightarrow$ $H(D)$ by

$$
S(x)(z)=\int_{0}^{z} x(t) d t \quad(x \in H(\mathbb{D}), z \in \mathbb{D}) .
$$

Referring to Duren [3] we have $S\left(H^{1 / 2}\right) \subseteq H^{1}$. Therefore, we can define an operator $S: H^{1 / 2} \rightarrow H^{1}$ which, looking at the closed-graph theorem, turns out to be continuous.

The reader may put the question whether $S$ is a compact linear operator or not. Assuming familiarity with harmonic functions one can show that the family $\left\{f_{r}\right\}_{0 \leq r<1}$, $f_{r}(z)=\frac{\left(1-r^{2}\right)^{2}}{(1-r z)^{4}}$ is bounded in $H^{1 / 2}$ and that the family $\left\{S f_{r}\right\}_{0 \leq r<1}$ has no convergent subsequence in $H^{\mathbf{1}}$. Therefore, $S$ cannnot be compact.

Now we use a composition operator to make $F_{\varphi}$ compact: Choose a function $\varphi \epsilon$ $H(D)$ with $\varphi(\mathbb{D}) \subseteq \mathbb{D}$. It induces a composition operator $C_{\varphi}$ by $C_{\varphi}(x)=x \circ \varphi(x \in$ $H(\mathbb{D})$ ). This is a linear operator and, using Littlewoods subordination theorem (see Duren [3]), in connection with the Harnack inequality (see Rudin [14]), we have its continuity on $H^{1}$. For $\varphi(0)=0$ we obtain the estimate $\left\|C_{\varphi}\right\| \leq 1$.

Compact composition operators have been analyzed by Shapiro and Taylor [16]. For example, $C_{\varphi}$ is nuclear and, therefore, compact, if the image of $\varphi$ is contained in a polygon which himself is contained in $D$ (see [16: Theorems 5.1 and 6.1]).

To reach the space $H^{1 / 2}$ we square the images of $C_{\varphi}$. This also guaranties the non-linarity of the resulting operator. We consider the operator

$$
T: H^{1} \longrightarrow H^{1 / 2} \text {. }
$$

It is clear that $T$ is defined. Let $G_{g}: H^{1} \rightarrow H^{1 / 2}\left(g \in H^{1}\right)$ be defined by $G_{g}(f)=$ $g f\left(f \in H^{1}\right)$. Using the Hölder inequality we have the equicontinuity of the family $\left\{G_{g}\right\}_{g \in A}$ for bounded $A \subseteq H^{1}$. Now the continuity of $T$ turns out by the triangle inequality.

We have arrived at the situation

$$
H^{1 / 2} \longrightarrow S H^{1} \longrightarrow C_{\varphi} H^{1} \longrightarrow T H^{1 / 2},
$$

and, if $B(r)$ denotes the ball of radius $r>0$ around zero in $H^{1 / 2}$,

$$
F_{\varphi, y}=T \circ C_{\varphi} \circ S+y \quad\left(y \in H^{1 / 2}\right)
$$

defines a continuous nonlinear compact operator $F_{\varphi, y}: B(r) \rightarrow H^{1 / 2}$. Since $r_{b}^{\prime}$ is algebraically semi-additive we have that $F=F_{\lambda}+F_{\varphi, y}$ condenses for $|\lambda|<1$. To use Theorem 11 we have to find requirements which provide $F(B(r)) \subseteq B(r)$.

Recognizing the definition of $F_{\varphi}$ we have that $S(B(r))$ is included in the ball of radius $\|S\| r^{2}$ around zero in $H^{1}$. Assuming $\varphi(0)=0$ we see that $F_{\varphi}(B(r)) \subseteq B\left(\|S\| r^{2}+\right.$ $\left.\|y\|_{1 / 2}\right)$. Therefore, supposing that

$$
r\left(|\lambda|^{1 / 2}+r\|S\|-1\right)+\|y\|_{1 / 2} \leq 0
$$


we get a solution of the equation $x-F x=y$ in $H^{1 / 2}$ where $\|x\|_{1 / 2} \leq r$ holds.

4.4 The spaces $L^{p}[0,1]$ for $p \in(0,1)$ and the space $S[0,1]$. Let $S[0,1]$ be the space of all measurable functions $f:[0,1] \rightarrow \mathbb{R}$. Then

$$
\|f\|=\int_{0}^{1} \frac{|f|}{1+|f|} d t \quad(f \in S[0,1])
$$

defines a $F$-norm and $S[0,1]$ is a complete $F$-normed space which is not locally bounded (see Jarchow [5]). The notion of convergence induced by (24) is convergence in measure, i.e.

$$
\lim _{n}\left\|f_{n}\right\|=0 \quad \Longleftrightarrow \quad \lim _{n} \mu\left\{x \in[0,1]|| f_{n}(x) \mid>\delta\right\}=0 \forall \delta>0
$$

for $\left(f_{\mathrm{n}}\right)_{\mathrm{n} \in \mathbb{N}} \subseteq S[0,1]$, at which $\mu$ denotes the Lebesgue measure on $[0,1]$.

Choose $p \in(0,1)$. If we define $L^{p}[0,1]=\left\{\left.f \in S[0,1]|| f\right|^{p}\right.$ is integrable $\}$, then the p-norm

$$
\|f\|_{p}=\int_{0}^{1}|f|^{p} d t \quad\left(f \in L^{p}[0,1]\right)
$$

induces a stronger notion of convergence as (25). The space $L^{P}[0,1]$ is not locally convex but it is locally bounded and complete (see Jarchow [5]). For $0<p \leq q<1$ we have the inclusions $L^{q}[0,1] \subseteq L^{p}[0,1] \subseteq S[0,1]$.

Riedrich (see $[12,13])$ has shown the admissibility of $L^{p}[0,1] \quad(0<p<1)$ and $S[0,1]$ :

For $n \in \mathbb{N}$ and $k \in\{1, \ldots, n\}$ let $I_{k, n}$ denote the intervall $\left[\frac{k-1}{n}, \frac{k}{n}\right]$ and $\chi_{k, n}$ it's characteristic function. For $n \in \mathbb{N}$ we define weights

$$
p_{k, n}(f)=\left(n \int_{I_{k, n}} f^{p} d t\right)^{1 / p} \quad(k \in\{1, \ldots, n\})
$$

for $0 \leq f \in L^{p}[0,1]$ and

$$
s_{k, n}(f)=\int_{I_{k, n}} \frac{f}{1+f} d t\left(\int_{I_{k, n}} \frac{1}{1+f} d t\right)^{-1} \quad(k \in\{1, \ldots, n\})
$$

for $0 \leq f \in S[0,1]$. The approximations are given by

$$
P_{n}(f)=\sum_{k=1}^{n} p_{k, n}(f) \chi_{k, n} \quad \text { and } \quad S_{n}(f)=\sum_{k=1}^{n} s_{k, n}(f) \chi_{k, n},
$$

for non-negative $f$, respectively. For general $f$ in $L^{p}[0,1]$ and $S[0,1]$ we define

$$
P_{n}(f)=P_{n}\left(f_{+}\right)-P_{n}\left(f_{-}\right) \quad \text { and } \quad S_{n}(f)=S_{n}\left(f_{+}\right)-S_{n}\left(f_{-}\right), .
$$


respectively, at which $f_{+}=\max \{f, 0\}$ and $f_{-}=\max \{-f, 0\}$.

For $n \in \mathbb{N}$ let $X_{n}$ denote the finite-dimensional subspace $\operatorname{span}\left\{\chi_{1, n}, \ldots, \chi_{n, n}\right\}$ of $L^{p}[0,1]$ or $S[0,1]$ : Riedrich [12] shows that the operators $P_{n}: L^{p}[0,1] \rightarrow X_{n}$ define an approximation sequence of equicontinuous retracts on $X_{n}$. In [13] an analogous result is given for the operators $S_{n}: S[0,1] \rightarrow X_{n}$.

Now we show that $\left(P_{n}\right)_{n \in \mathbb{N}}$ satisfies the requirements of Corollary 1:

Estimate (3) in Section 2 of [12] yields for all $0<p<1$ and $\sigma>0$ the existence of a $\lambda=\lambda(p, \sigma)$ such that

$$
|t-s|^{p} \leq \lambda\left|t^{p}-s^{p}\right|+\sigma\left|t^{p}+s^{p}\right| \quad \text { for all } t, s \geq 0
$$

and Riedrichs shows that

$$
\left\|P_{n}(f)-P_{n}(g)\right\|_{p} \leq 2 \lambda\|f-g\|_{p}+2 \sigma\left(\|f\|_{p}+\|g\|_{p}\right) . \text { for all } f, g \in L^{p}[0,1]
$$

at which $\lambda$ also depends on $p$ and $\sigma$ only. Using (27) we have that for every bounded set $M \subseteq L^{p}[0,1]$ the set $\cup_{n \in \mathbb{N}} P_{n}(M)$ is bounded and $\left\{P_{n}\right\}_{n \in \mathbb{N}}$ is uniformly equicontinuous on $M$. Therefore, suppositions (i) und (ii) of Corollary 1 hold.

Concerning supposition (iii) of Corollary 1 we have to use $\left\{2^{n} \mid n \in \mathbb{N}\right\}$ to index the approximation sequence. Since $\left.P_{n}\right|_{X_{n}}=I$ we have $P_{m} \circ P_{n}=P_{\min \{m, n\}}(m \geq n)$. In case of $m<n$ we have for $k \in\{1, \ldots, m\}, m=2^{v}$ and $n=2^{v+w}$ the calculation

$$
\begin{aligned}
p_{k, m}\left(\left(P_{n} f\right)_{ \pm}\right) & =p_{k, m}\left(P_{n} f_{ \pm}\right) \\
& =\left(m \int_{I_{k, m}}\left(\sum_{j=2 \omega(k-1)+1}^{2^{\omega} k}\left(n \int_{I_{j, n}} f_{ \pm}^{p} d t\right)^{1 / p} \chi_{j, n}\right)^{p} d t^{\prime}\right)^{1 / p} \\
& =\left(m \int_{I_{k, m}} \sum_{j=2^{\omega}(k-1)+1}^{2^{\omega} k}\left(n \int_{I_{j, n}} f_{ \pm}^{p} d t\right) \chi_{j, n} d t^{\prime}\right)^{1 / p} \\
& =\left(m \int_{I_{k, m}} f_{ \pm}^{p} d t^{\prime}\right)^{1 / p} \\
& =p_{k, m}\left(f_{ \pm}\right)
\end{aligned}
$$

for the weights and, again, $P_{m} \circ P_{n}=P_{\min \{m, n\}}$.

Since the suppositions of Corollary 1 hold we have that every continuous map with bounded image which is $\frac{1}{2}$-condensing with respect to $r_{p}$ is approximation-compact with respect to 0 and $\left(P_{n}\right)_{n \in \mathbb{N}}$.

An analoguous result holds for $\left(S_{n}\right)_{n \in \mathbb{N}}$ using a little bit more argumentation since there is no estimate like (27). For the detailed proof of suppositions (i) - (iii) of Corollary 1 we refer to [10] since it is only technical.

We have arrived at the obligatory fixed-point theorems. 
Theorem 12. Let $\theta \neq \Omega \subseteq L^{p}[0,1]$ or $\emptyset \neq \Omega \subseteq S[0,1]$ be a shrinkable neighbourhood of zero, and let $F: \bar{\Omega} \rightarrow \bar{\Omega}$ be a continuous map with bounded image which is $\frac{1}{2}$-condensing with respect to $r_{p}$. In case of the space $S[0,1]$ the maps $t F$ have assumed to be $\frac{1}{2}$-condensing for $t \in(0,1)$. Then $F$ has a fixed point.

Proof. We can use the generalized degree since the image of $F$ is bounded and the approximations map bounded sets into bounded sets.

For every $f \in L_{p}[0,1]$ and $t \in(0,1)$ we have the equations $P_{n}(t f)=t P_{n}(f)$ and $\|t f\|_{p}=|t|^{p}\|f\|_{p}$ which provide that $t F$ is $\frac{1}{2}$-condensing. This completes the proof

Now we deal with the motivation of the notion of $\psi$-condensing maps and the measure $r_{b}$. Riedrichs proof of $(26)$ is indirect, but we have to look at two functions

$$
\varphi_{\sigma}(u)=(1-u)^{p}-\sigma\left(1+u^{p}\right) \quad \text { and } \quad \psi(u)=1-u^{p} \quad(0 \leq u \leq 1)
$$

with a parameter $\sigma>0$. We have to find a constant $\lambda>0$ such that

$$
\varphi_{\sigma}(u) \leq \lambda \psi(u) \quad(0 \leq u \leq 1)
$$

Now we take a close look at

$$
a(u):=\frac{(1-u)^{p}}{1-u^{p}} \quad \text { and } \quad b_{\sigma}(u):=-\sigma \frac{1+u^{p}}{1-u^{p}} \quad(0 \leq u<1)
$$

and see that

$$
H(\sigma)=\max _{0 \leq u<1}\left(a(u)+b_{\sigma}(u)\right)
$$

is a possible value for $\lambda$.

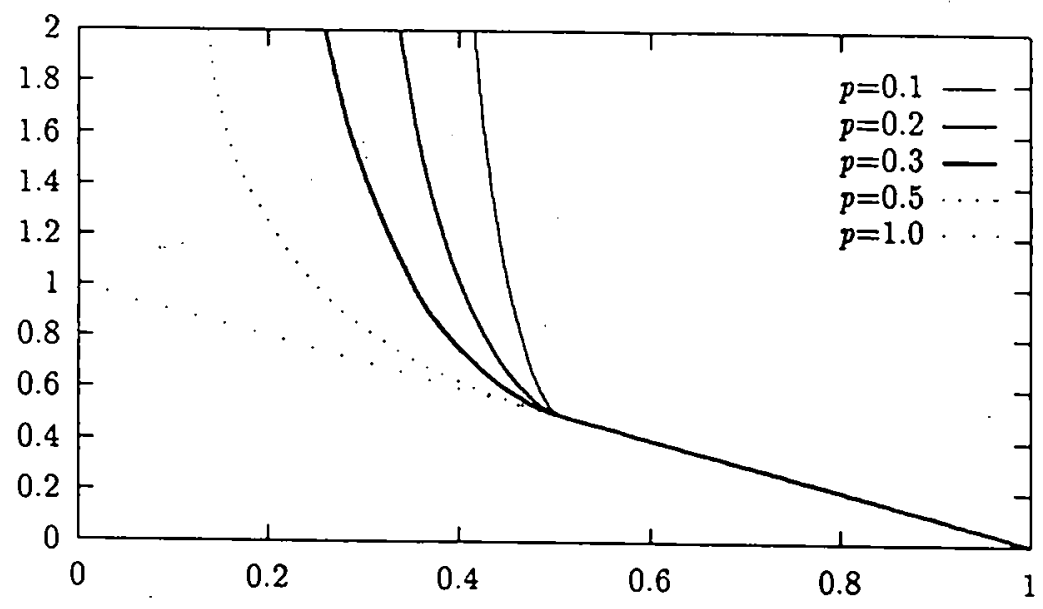

Figure 1: $H=H(\sigma)$ for different $p \in(0,1]$

Therefore, we can write (27) in the form

$$
\left\|P_{n}(f)-P_{n}(g)\right\|_{p} \leq 2 H(\sigma)\|f-g\|_{p}+2 \sigma\left(\|f\|_{p}+\|g\|_{p}\right) \quad(\sigma \in(0,1])
$$


at which $f, g \in L_{p}[0,1]$ and $n \in \mathbb{N}$.

Let $\emptyset \neq \Omega \subseteq L^{p}[0,1]$ be open and bounded. Define

$$
\hat{H}(\Omega, t, \sigma)=2 H(\sigma) t+4 \operatorname{diam}(\Omega) \sigma \quad(t \in[0, \infty), \sigma \in(0,1]) .
$$

Using (28) we get for $\left(x_{n}\right)_{n \in \mathbb{N}} \subseteq \bar{\Omega}$ and $r_{b}$ the estimate

$$
r_{b}\left(\left\{P_{n} x_{n} \mid n \in \mathbb{N}\right\}\right) \leq \widehat{H}\left(\Omega, r_{b}\left(\left\{x_{n} \mid n \in \mathbb{N}\right\}\right), \sigma\right) \quad(\sigma \in(0,1]) .
$$

We can define

$$
\psi_{\Omega}(t)=\inf \{\hat{H}(\Omega, t, \sigma) \mid 0<\sigma \leq 1\} \quad(0 \leq t<\infty)
$$

since $\hat{H}(\Omega, t, \cdot)$ is continuous and bounded from below by 0 . Therefore, we have

$$
r_{b}\left(\left\{P_{n_{k}} x_{n_{k}} \mid k \in \mathbb{N}\right\}\right) \leq \psi_{\Omega}\left(r_{b}\left(\left\{x_{n_{k}} \mid k \in \mathbb{N}\right\}\right)\right)
$$

for every subsequence $\left(x_{n_{k}}\right)_{k \in \mathbb{N}}$ of $\left(x_{n}\right)_{n \in N}$. The function $\psi_{\Omega}$ is a strictly monotonous increasing one and $H(\sigma)>0(0<\sigma<1)$ provides that $\psi_{\Omega}(t)=0$ if and only if $t=0$. Therefore, $\psi_{\Omega}^{-1}$ is a strictly monotonous increasing function, too, and, using Lemma 2 , every continuous map $F: \bar{\Omega} \rightarrow L_{p}[0,1]$ which is $\psi_{\Omega}^{-1}$-condensing with respect to $r_{b}$ is approximation-compact.

Theorem 13. Let $\emptyset \neq \Omega \subseteq L^{p}[0,1]$ be an open and bounded shrinkable neighbourhood of zero. Suppose $F: \bar{\Omega} \rightarrow \bar{\Omega}$ is continuous and $\psi_{\Omega}^{-1}$-condensing with respect to $r_{b}$, at which $\psi_{\Omega}^{-1}$ is given by (30). Then $F$ has a fixed point. to $r_{b}$

Proof. We only note that the maps $t F(t \in(0,1))$ are $\psi_{\Omega}^{-1}$-condensing with respect

Remark. Since $H$ has a singularity at zero, we have

$$
\frac{\psi_{\Omega}(t)}{t}=\inf _{0<\sigma \leq 1}\left(2 H(\sigma)+\frac{4 \sigma \operatorname{diam}(\Omega)}{t}\right) \rightarrow+\infty \quad \text { for } t \rightarrow 0+
$$

Therefore, only using (27) to get an estimate as (31), we cannot make a statement concerning maps that are $C$-condensing with respect to $r_{b}$, at which $C$ is a constant.

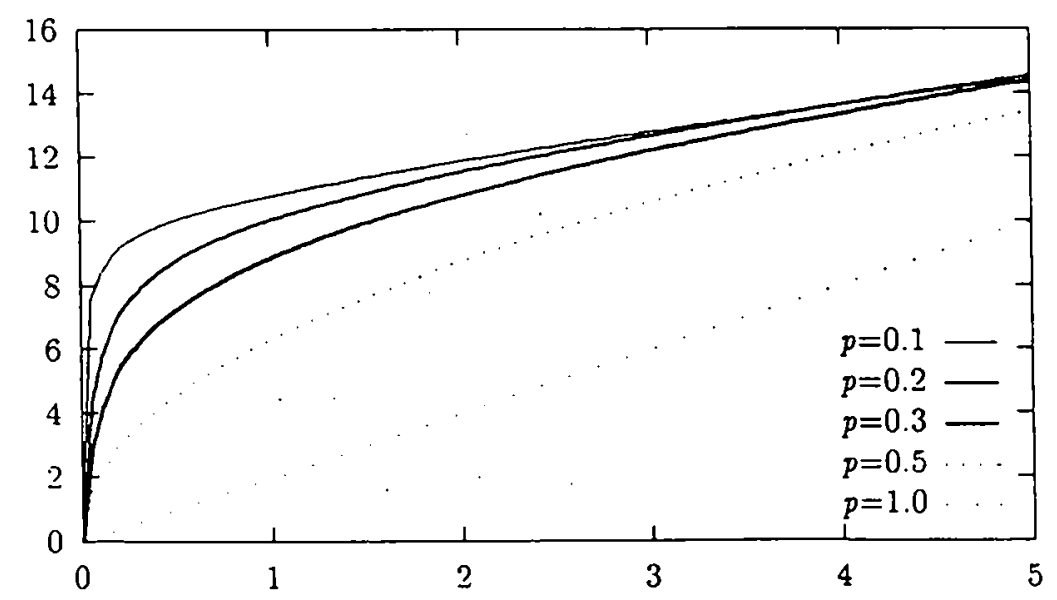

Figure 2: $\psi_{\Omega}=\psi_{\Omega}(t)$ for different $p \in(0,1]$ and $\operatorname{diam}(\Omega)=5$ 

of $\Omega$.

The definition (29) of $\widehat{H}$ tells us that we get the same figure for any other diameter

Remark. Both figures have been produced by using the computer program Mathematica for approximation of discrete date and are only claimed to be illustrations.

Acknowledgement. The author is grateful to Prof. Dr. Winfried Kaballo for formulating the problem and supporting it's solution in the graduate work [10]. Thanks to Dr. habil. Alexandru Aleman for indications concerning Hardy spaces. And, last but not least, special thanks to Prof. Dr. Thomas Riedrich for the interest in the authors work and for the motivation of this paper.

\section{References}

[1] Alex, H., Hahn, S. and L. Kaniok: The fixed point index for noncompact mappings in non locally convex topological vector spaces. Comm. Math. Univ. Carolinae 32 (1994), 249 257.

[2] Browder, F. E. and W. V. Petryshyn: Approximation methods and the generalized topological degree for nonlinear mappings in Banach spaces. J. Func. Anal. 3 (1968), 217 245.

[3] Duren, P. L.: Theory of $H^{p}$-Spaces. New York: Academic Press 1970.

[4] Hahn, S. and K. F. Pötter: Über Fixpunkte kompakter Abbildungen in topologischen Vektorräumen. Studia Math. 50 (1974), $1-16$.

[5] Jarchow, H.: Locally Concex Spaces. Stuttgart: B. G. Teubner 1981.

[6] Kaballo, W.: Zum Abbildungsgrad in Hausdorffschen topologischen Vektorräumen. Man. Math. 2 (1973), 209 - 216.

[7] Kayser, G.: Zur relativen Drehung in Hausdorffschen topologischen Vektorräumen. Wiss. Z. Techn. Univ. Dresden 23 (1973), $335-340$.

[8] Krauthausen, C.: On the theorems of Dugundji and Schauder for certain nonconvex spaces. Mat. Balkanica 4 (1974), 365 - 369.

[9] Landsberg, M.: Lineare topologische Räume, die nicht lokalkonvex sind. Math. 2. 65 (1956), $104-112$.

[10] Okon, T.: Ein Abbildungsgrad für approximationskompakte Störungen der Identität in nicht notwendig konvexen Räumen. Diplomarbeit. Dortmund: Universität 1994.

[11] Priwalow, I. I.: Randwerte analytischer Funktionen. Berlin: Dt. Verlag Wiss. 1956.

[12] Riedrich, Th.: Die Räume $L^{p}(0,1)(0<p<1)$ sind zulässig. Wiss. Z. Techn. Univ. Dresden 12 (1963); 1149 - 1152.

[13] Riedrich, Th.: Der Raum $S(0,1)$ ist zulässig. Wiss. Z. Techn. Univ. Dresden 13 (1964), $1-6$.

[14] Rudin, W.: Real and Complex Analysis. New York: McGraw-Hill 1966.

[15] Sadovskii, B. N.: Limit-compact operators, measures of non-compactness, and condensing operators. Russ. Math. Surveys 27 (1972), 85 - 155.

[16] Shapiro, J. H. and P. D. Taylor: Compact, nuclear and Hilbert-Schmidt composition operalors on $H^{2}$. Indiana Math. J. 23 (1973), $471-496$. 
[17] van der Bijl, J., Dobrowolski, T., Hart, K. P. and van der Mill, J.: Admissibility, homeomorphism extension and the $A R$-property in topological linear spaces. Top. and its Appl. 48 (1992), $63-80$.

[18] van der Bijl, J. and J. van der Mill: Linear spaces, absolute retracts, and the compact extension property. Proc. Amer. Math. Soc. 104 (1988), 942 - 952.

[19] Yanagihara, N.: Multipliers and linear functionals for the class $N^{+}$. Trans. Amer. Math. Soc. 180 (1973), $449-461$.

Received 30.12.1994; in revised form 30.05.1995 